\title{
Haze and Cloud structure of Saturn's North Pole and Hexagon Wave from Cassini/ISS imaging
}

\author{
J.F. Sanz-Requena ${ }^{\mathrm{a}}$, S. Pérez-Hoyos ${ }^{\mathrm{b}}$, A. Sánchez-Lavega ${ }^{\mathrm{b}}$, A. Antuñano ${ }^{\mathrm{b}}$, Patrick G.J. \\ Irwin $^{c}$ \\ a) Departamento de Ciencias Experimentales. Universidad Europea Miguel de \\ Cervantes, Valladolid, Spain. \\ b) Departamento de Física Aplicada I, Escuela Técnica Superior de Ingeniería, \\ Universidad del País Vasco, Bilbao, Spain \\ c) Atmospheric, Oceanic and Planetary Physics, University of Oxford, Clarendon \\ Laboratory, Parks Road, Oxford OX1 3PU, UK
}

\section{Abstract}

In this paper we present a study of the vertical haze and cloud structure in the upper two bars of Saturn's Northern Polar atmosphere using the Imaging Science Subsystem (ISS) instrument onboard the Cassini spacecraft. We focus on the characterization of latitudes from $53^{\circ}$ to $90^{\circ} \mathrm{N}$. The observations were taken during June 2013 with five different filters (VIO, BL1, MT2, CB2 and MT3) covering spectral range from the $420 \mathrm{~nm}$ to 890 $\mathrm{nm}$ (in a deep methane absorption band). Absolute reflectivity measurements of seven selected regions at all wavelengths and several illumination and observation geometries are compared with the values produced by a radiative transfer model. The changes in reflectivity at these latitudes are mostly attributed to changes in the tropospheric haze. This includes the haze base height (from $600 \pm 200$ mbar at the lowest latitudes to $1000 \pm 300 \mathrm{mbar}$ in the pole), its particle number density (from $20 \pm 2$ particles $/ \mathrm{cm}^{3}$ to $2 \pm 0.5$ particles $/ \mathrm{cm}^{3}$ at the haze base) and its scale height (from $18 \pm 0.1 \mathrm{~km}$ to $50 \pm 0.1$ $\mathrm{km})$. We also report variability in the retrieved particle size distribution and refractive indices. We find that the Hexagonal Wave dichotomizes the studied stratospheric and tropospheric hazes between the outer, equatorward regions and the inner, Polar Regions. This suggests that the wave or the jet isolates the particle distribution at least at tropospheric levels.

\section{Keywords:}

Atmospheres, structure; Saturn; Saturn, atmosphere; Radiative transfer 


\section{Highlights}

- We study the North Polar Region of Saturn based on Cassini ISS images.

- The changes in reflectivity are attributed to changes in the tropospheric haze.

- The Hexagonal Wave dichotomizes the latitudes: the outer, and the inner regions.

- Retrieved atmospheric model are in agreement with dynamical models of the polar region.

\section{Introduction}

The North Pole of Saturn is a region where we find unique atmospheric features as we move from the Equator to higher latitudes. Examples of these are the Hexagon Wave, a fast eastward jet, a large number of "puffy" clouds mixed with local cyclonic and anticyclonic vortices, and the polar vortex, which includes a fast eastward jet (Godfrey, 1988; Fletcher et al., 2008; Sánchez-Lavega et al., 2014; Antuñano et al., 2015, 2018).

Saturn's Hexagon Wave is a singular feature centered close to $75^{\circ} \mathrm{N}$ planetocentric latitude (all latitudes in this work will be given as planetocentric). The feature was discovered in 1980-1981 in images taken during Voyager 1 and 2 flybys (Godfrey, 1988). In 1991 the Hexagon Wave was observed again after a long polar Saturnian winter by the Hubble Space Telescope (Caldwell et al., 1993) and, afterwards, even with ground-based telescopes (Sánchez-Lavega et al., 1993). This showed that it is a long-lived atmospheric feature. This was confirmed one Saturnian year after its discovery with the arrival of the Cassini mission in 2004. Since 2012, the Hexagon Wave has also been clearly and routinely discernible in images captured by groundbased telescopes and amateur astronomers (Sanchez-Lavega et al., 2014). In short, the Hexagonal Wave has been studied since 1980 until 2017 and due to its stable rotation period, it has been proposed that it could track the rotation rate of the planet (SanchezLavega et al., 2014).

Since the arrival of Cassini at Saturn in 2004, the Hexagon Wave was observed in 2006-2007 in the thermal infrared using the Visible and Infrared Mapping 
Spectrometer (VIMS) (Brown et al., 2004) at $5 \mu \mathrm{m}$, while the North Pole was still in winter with little or no solar illumination. Since 2008, the Hexagon Wave has been observed in reflected sunlight throughout the remaining years with the Cassini Image Science Subsystem (ISS) cameras (Antuñano et al. 2015; Sayanagi et al., 2017). It is well known that Saturn shows an intense zonal circulation at the cloud top level formed by a system of jet streams that alternate their east-west direction depending on latitude (see e.g. García-Melendo et al., 2011 for a review). Saturn's North Pole Hexagon Wave encloses a fast $\left(\sim 100 \mathrm{~ms}^{-1}\right)$ eastward jet at latitude $75.8^{\circ} \mathrm{N}$ (Godfrey, 1988).

The Hexagon Wave has been proposed to be a vertically-trapped Rossby wave (Allison et al., 1990; Sánchez-Lavega et al., 2014) located in a range of latitudes from $75.3^{\circ}$ to $76.3^{\circ} \mathrm{N}$. It has a planetary scale, with a length of approximately 30,000 kilometers centered at the North Pole of the planet and with wave number six. It could possibly result from an instability of the strong jet in its interior (Barbosa-Aguiar et al., 2010; Morales-Juberías et al., 2011). Nothing like a polygonal wave has been found in any other planet of the Solar System, except some features in the eye of Earth hurricanes (Kossin and Schubert, 2001). Jupiter for example, displays a complex structure of polar waves, but with no polygonal shapes (Sánchez-Lavega et al., 1997; Barrado-Izagirre et al., 2008).

Another interesting atmospheric features at high latitudes are the intense cyclonic polar vortices. These vortices were detected in Saturn's poles in the Cassini era, extending in latitudes from $88.5^{\circ}$ to $90^{\circ}$ north and south (Sánchez-Lavega et al., 2006; Dyudina et al., 2008, Fletcher et al., 2008; Baines et al., 2009; Dyudina et al., 2009; Sayanagi et al., 2013). Both vortices are enclosed by a very fast $\left(\sim 140-160 \mathrm{~ms}^{-1}\right)$ eastward jet at latitude $88.5^{\circ}$ (Antuñano et al, 2015, Sayanagi et al., 2017).

The dynamics at Saturn polar latitudes is particularly appealing for a number of reasons (Sayanagi et al., 2016). For example, the presence of intense polar vortices is particularly intriguing, as Juno mission has not revealed anything similar in Jupiter's atmosphere. Given the tilt of the rotation axis, Saturn's poles suffer long periods of darkness during the polar winter and, although seasonal changes have been reported at the stratosphere in both the temperature field and hydrocarbon distribution (Fletcher et al., 2015), the dynamical features described above were able to survive from one season to the next without noticeable changes. This suggests deeply rooted structure that have been suggested to be linked with the internal rotation period (Sánchez-Lavega et al., 2014). 
In order to understand such lively atmospheric dynamics it is essential to know the vertical distribution of haze and clouds, which are used as its tracers. This also requires the knowledge of the physical and optical properties of the cloud and haze particles in the stratosphere and upper troposphere of Saturn. Our current understanding of Saturn's clouds and hazes is constrained by several decades of remote sensing data. Thus, we can divide Saturn into three large separate latitudinal regions (Pérez-Hoyos et al., 2005) and an overall vertical structure formed by a thin stratospheric haze and a denser tropospheric haze, both above a thick cloud layer (Pérez-Hoyos et al., 2005; Roman et al. 2013). An excellent review of the state of the art regarding Saturnian hazes and clouds can be found in West et al. (2009). Particles in the uppermost stratospheric haze are commonly assumed or obtained to be small (tenths of microns), but most likely they are not spherical in view of results regarding Jupiter's atmosphere (West et al., 1991). The deepest detectable particle layer in visible wavelengths is putatively formed by ammonia ice and has uncertain properties that require longer and more penetrating wavelengths than those used in this work (Fletcher et al., 2008).

Most of the information contained in the visible light scattered by the atmosphere comes from the upper tropospheric cloud, whose variable cloud-top pressure strongly dominates the brightness of the planet at near infrared methane bands (Roman et al., 2013). While very often assumed to be spherical (Karkoschka et al., 2005), it is known that their phase function does not match this shape and some efforts have been performed in order to retrieve a more accurate description (Tomasko and Doose, 1984; Pérez-Hoyos et al., 2016). This general picture of the vertical structure of particles is able to explain also the structure of particle features of the atmosphere (Roman et al., 2013), such as vortices (del Río-Gaztelurrutia et al., 2010) or the periodic giant storms (Sanz-Requena et al., 2011).

However, no previous work has been able to study the northern polar region of Saturn in the context of a radiative transfer model of the reflected sunlight. The orbits by the Cassini orbited allowed an unprecedented spatial resolution over the pole and provided access to a region of the planet that had been unexplored so far. For example, Cassini ISS polarization images of the region (West et al., 2015) revealed changes in the aerosol properties inside and outside the Hexagon but a quantitative analysis is still missing. Retrievals of the properties of the upper tropospheric cloud and stratospheric hazes in the polar region has important implications for studies of the dynamical coupling between the stratosphere and troposphere (Del Genio et al., 2009), and the 
137 composition and chemistry in the stratosphere (Fouchet et al., 2009). Particularly important is to understand how singular dynamical features observed at tropospheric level, like the already mentioned hexagon and its jet and the polar vortex, penetrate and leave their imprint at the stratospheric level. Comparison with dynamical conditions in the stratosphere, via thermal winds and static stability conditions from temperature data can shed light on vertical wave propagation in the polar stratosphere, for example (Sánchez-Lavega et al, 2017). The long-term unchanging behavior observed in the tropospheric jets and upper cloud morphology (Antuñano et al., 2017), but with changes in the temperature and ortho-para hydrogen distribution could influence the cloud structure (Fletcher et al., 2016). The aerosol vertical distribution has a profound influence on the heating and cooling rates in the stratosphere where the insolation seasonal cycle can be studied in detail (Sylvestre et al., 2015; Fletcher et al. 2016).

The goal of this paper is to evaluate the structure of the clouds and hazes and the distribution of aerosols and particles in the upper troposphere and lower stratosphere of Saturn's North Pole and its surrounding area, using Cassini Image Science Subsystem (ISS) data sets in different filters. Baines et al. (2009) modeled the properties of clouds below this level (altitude range 2-5 bar) using Cassini-VIMS spectra in the $5 \mu \mathrm{m}$ wavelength range. In order to avoid complications arising from the temporal evolution of the atmosphere, we are restricted to a snapshot around 2013 when the observations used here were made. In this paper we analyze the variation of the absolute reflectivity with observation/illumination geometries at filters from $420 \mathrm{~nm}$ (VIO) to the deep methane band at $890 \mathrm{~nm}$ (MT3). We intend to build the simplest atmospheric model that concurrently satisfies the observed reflectance and contrast at multiple wavelengths and viewing angles. By exploiting the wavelength and geometric dependencies in the radiative transfer equations, we will be able to constrain different atmospheric parameters, mostly of the tropospheric haze around the tropopause level

The paper is organized as follows: Section 2 is devoted to a short description of the observations used in this work. Section 3 covers the radiative transfer model, including the description of the vertical cloud structure model and the a priori assumptions. Results are presented and discussed in Section 4. We also present in this section the sensitivity to the model parameters. The results are discussed in Section 5 in terms of the local dynamics. A summary of the main conclusions of this work is presented in Section 6. 
170

171

172

173

174

175

176

177

178

179

180

181

182

183

184

185

186

187

188

189

190

191

192

193

194

195

196

197

198

199

200

201

\section{Observations}

\subsection{Description of the observations}

In this study we have used 40 images taken with the Imaging Science Subsystem (ISS) onboard the Cassini spacecraft. A full description of the ISS instrument can be found in Porco et al. (2004). We show in Figure 1 a representative set of the Saturn images used in this work, shown as polar projections. Images were taken from June 3 to June 26, 2013 with five different filters, all pertaining to ISS 2083. In Table 1 we show the image identification number, date of observation, filter name, date and time of image acquisition, the sub-spacecraft planetocentric latitude $\mathrm{B}$, the sub-solar planetocentric latitude B' and the phase angle $\alpha$, defined as the observer-planet-Sun angle (SánchezLavega, 2011). The observing period was close to Saturn's Northern Hemisphere summer solstice (West et al., 2009). All the images were navigated using PLIA software (Hueso et al., 2010) and photometrically calibrated to absolute reflectivity using Cassini Imaging Science Subsystem CALibration software (CISSCAL v3.6) described by Porco et al. (2004) and West et al. (2010).

The selection includes two broad-band filters at short wavelengths, violet (VIO, $420 \mathrm{~nm})$ and blue (BL1, 451nm), as well as three near-infrared narrow-band filters matching methane bands MT2 $(727 \mathrm{~nm})$, MT3, $(890 \mathrm{~nm})$ and an intermediate continuum filter (CB2, $752 \mathrm{~nm}$ ). Transmission curves can be found in Porco et al. (2004). A representative image of Saturn in each of the five filters is shown in Figure 1. VIO and BL1 filters (Fig 1a and Fig. 1b) are sensitive to the colors of the clouds and hazes, which provide compositional information and scattering properties at various heights. and to Rayleigh scattering by the $\mathrm{H}_{2}$ and $\mathrm{He}$ atmosphere. In images taken with the filters BL1 and VIO the interior of the Hexagon Wave is brighter than the exterior and a gradual darkening is observed as we approach the pole.

The methane-band filters (MT2, intermediate absorption, and MT3, deep absorption, Figures 1c and 1e) are sensitive to the upper tropospheric haze layer (PérezHoyos et al., 2016). These filters sense particles at altitudes between 60 mbar and 250 mbar (West et al., 2009; García-Melendo et al., 2009, 2011). The continuum band filter (CB2, $752 \mathrm{~nm}$, Fig.1d) is sensitive to an intermediate region of the tropospheric haze (altitudes between 350 mbar and 700 mbar, García-Melendo et al., 2009, 2011), down to the top of the tropospheric cloud deck, assumed here to be located at 1.4 bar and 
202 putatively formed of ammonia ice (Sanchez-Lavega, 2011). At the wavelengths of the

203 CB2 filter, sunlight penetrates the hazes and Saturn's North polar region reveals a

204

205

206

207

208

209

210

211

212

213

214

215

216

217

218

219

220

221

222

223

224

225

226

227

228

229

230

231

232

233 variety of dynamical features, such as an ample field of 'puffy clouds' with sizes between 10 and $500 \mathrm{~km}$, and cyclones and anticyclones of different sizes and lifetimes distributed in the region (Antuñano et al., 2018). These features are usually not observable at the wavelengths of the VIO, MT2 and MT3 filters.

\subsection{Data clustering and selection}

To divide the range of latitudes studied here (from $53^{\circ}$ to $90^{\circ} \mathrm{N}$ ) into a manageable number of sets, we used a K-means supervised clustering algorithm (MacQueen, 1967) as in Pérez-Hoyos et al. (2012). We initially populated seven clusters manually for each phase angle. This was done to avoid differences in viewing and illumination geometry that are sometimes greater than the reflectivity changes from one cluster to another at a given geometry. Then, the algorithm classifies the rest of the data assigning each instance to the nearest group. Each cluster center is updated to be the average of its constituent members. The algorithm converges when no more changes are possible in the allocation of clusters.

We show in Fig. 1f, the whole range of latitudes divided into 7 families. Regions 1, 2 and 3 correspond to latitudes equatorward of the Hexagonal Wave. Region 1 covers a bright zone from $53^{\circ}$ to $57^{\circ} \mathrm{N}$, region 2 a dark belt from $57^{\circ}$ to $65^{\circ} \mathrm{N}$ and region 3 a zone again from $65^{\circ}$ to $73^{\circ} \mathrm{N}$. Region 4 corresponds to latitudes $73^{\circ}$ to $77^{\circ} \mathrm{N}$ that encloses the Hexagonal Wave (Sánchez-Lavega, et al., 2014). Regions 5, 6, and 7 are associated with latitudes closer to the North Pole, comprising $77^{\circ}$ to $90^{\circ} \mathrm{N}$. Region 5 is a polar region which shows no cloud structure in the continuum CB2 filter, while region 6 is composed by samples with clouds bright at CB2. Finally, region 7 is the polar vortex itself, which looks dark at all filters and dates. These regions correspond to areas of different mean zonal flow vorticity (cyclonic-anticyclonic) according to the wind velocity profile shown in Figure 2 (García-Melendo et al., 2011; Antuñano et al., 2015, 2018). In Figure 3, we show an example of the actual data in Region 3 for a couple of filters, together with their average and standard deviation. This reduced data set will be used to retrieve the model parameters.

\section{Methodology}




\subsection{Radiative Transfer code}

In this work we have used the radiative transfer code and retrieval suite NEMESIS (Non-Linear Optimal Estimator for Multivariat ESpectral AnalySIS) developed by the Oxford team (Irwin et al., 2008). The version of NEMESIS used here is based a doubling-adding scheme that assumes a plane-parallel atmosphere. We include Rayleigh scattering due to the mixture of $\mathrm{H}_{2}$ and $\mathrm{He}$, which is very important in VIO and BL1 filters, as well as the absorption due to $\mathrm{CH}_{4}$, which is essential at certain filters like MT2 and MT3 (Sayanagi et al., 2016).

The goal of our modeling is to reproduce the observed dependence of absolute reflectivity with geometry (location on the disk and solar phase angle) for all wavelengths at the same time. We assume that the vertical structure does not change during the observations, hence the importance of focusing in a relatively narrow time span (Pérez-Hoyos et al., 2005). In order to reproduce all observations simultaneously, NEMESIS uses the optimal estimator scheme to find the most likely model that best accounts for the observations.

\subsection{Vertical cloud structure model}

In previous work (Pérez-Hoyos et al., 2005; Sanz- Requena et al. 2012; Pérez-Hoyos et al., 2016), Saturn's atmosphere was initially considered to be divided into three distinct layers of particles: an upper stratospheric haze (at 10s of mbar), a tropospheric haze in the upper troposphere (at a few 100s of mbar) and a bottom cloud at the ammonia condensation level. This approach has been found to be complex enough to account for spectral and geometrical variations of absolute reflectivity at visible wavelengths (Karkoschka and Tomasko, 1993, 2005). The vertical structure assumed in the present work is shown in Figure 4 and a complete list of the parameters, free and fixed, can be found in Table 2. It must be noted that NEMESIS assumes an a priori model from which we iterate until convergence to a satisfactory fit. This process is determined by the parameters assumed to be free but also by their a priori error bars. The election of free and fixed parameters was mostly based on a previous analysis and results by PérezHoyos et al., 2016. In short, we fixed the parameters that have little or none impact on the observed radiances at the filters of interest. Most of the influential parameters are found to be related with the tropospheric haze and hence this is the layer described in 
265

266

267

268

269

270

271

272

273

274

275

276

277

278

279

280

281

282

283

284

285

286

287

288

289

290

291

292

293

294

295

296

more detail by the model. Most of the a priori assumptions do not alter the retrieved free parameters, but a few comments will be discussed later on for some specific cases.

The uppermost particle layer corresponds to the stratospheric haze. We assume that this layer is located between pressures $P_{1}=1$ mbar and $P_{2}=100$ mbar (Pérez-Hoyos et al., 2016) and its particle density is constant with height (Pérez-Hoyos et al., 2005). The only free parameter of this layer, the stratospheric haze, is its total optical thickness $\left(\tau_{s t r}\right)$. As a starting point we have taken the value of $\tau_{s t r}=0.03 \pm 0.02$ (at $0.8 \mu \mathrm{m}$ ) given by Pérez-Hoyos et al. (2016) for the EZ, (Equatorial Zone) at $0.89 \mu \mathrm{m}$. As in previous work (Pérez-Hoyos et al., 2005, Sanz-Requena et al., 2012), the phase function is described by the Mie formalism, which requires a refractive index with real part $\left(m_{r}\right)$ and imaginary parts $\left(m_{i}\right)$. We assume for the real part a value of $m_{r}=1.43$ and for the imaginary part $m_{i}=10^{-3}$, both constant with wavelength and similar to the observed indices of ammonia ice at these wavelengths (Martonchik et al., 1984). In this case we employed the log-normal particle size distribution of Hansen and Travis (1974) (PerezHoyos et al., 2016) with an effective radius $\left(r_{\text {eff }}\right)$ of $0.15 \mu \mathrm{m}$ and an effective variance $\left(\sigma_{e f f}\right)$ of 0.1 (as in Sanz-Requena et al., 2012, and Ortiz et al., 1996), although these parameters are found to have little impact in the retrievals compared with others.

The intermediate layer corresponds to the tropospheric haze. In our model, the profile for the tropospheric haze is represented by a variable base height, a peak particle number density and scale height in terms of the atmospheric scale height. In this case, for the base altitude pressure $\left(P_{b o t}\right)$ we have taken 700 mbar as the a priori value following the result of $625 \pm 96$ mbar found by Roman et al., 2013. This second layer is allowed (and in fact found to) overlap with the upper haze. For the particle number density $(N)$ we have taken as reference the one found by Pérez-Hoyos et al. (2016) at a latitude $60^{\circ} \mathrm{N}$ of $10 \pm 5$ particles $/ \mathrm{cm}^{3}$. As an initial value for the height scale of the particles we have used the gas scale height $\left(\mathrm{H}_{\mathrm{g}}\right)$ in Saturn's atmosphere $(35 \pm 10 \mathrm{~km}$ at 100 mbar, Sánchez-Lavega, 2011).

For the tropospheric haze, we used Mie theory for the particle phase function calculations. In this work, we have a relatively narrow range of phase angles used but it is centered around some features specific of spherical particles. In order to remove these features, while retaining the ability to compute the particle cross section as a function of wavelength, we used in all calculations a fit to the expected Mie phase function using a 
297 Henyey-Greenstein phase function. A comparison with previous values for the Mie phase function and the Henyey-Greenstein fit will be provided in the corresponding section. Therefore, we also require as initial parameters the effective radius and variance of the size distribution. The values of initial $r_{\text {eff }}=1.5 \pm 0.5$ and $\sigma_{\text {eff }}=0.1 \pm 0.1$ were taken from Ortiz et al. (1996).

The refractive indices as a function of wavelength are also left as free parameters. In this model, the code of the real part of the refractive index spectrum is calculated with the Kramers-Kronig's relation (Lucarini et al, 2005) from an a priori value of $m_{r}=1.43$ expected for ammonia at a reference wavelength of $0.89 \mu \mathrm{m}$. (Martonchik et al., 1984), while the imaginary part is left as a free parameter. We use as initial value at all wavelengths $m_{i}=10^{-3}$ (Pérez-Hoyos et al., 2005).

Let's focus on the standard gamma distribution used in this work for the tropospheric particles, as in Roman et al. (2013). This can be expressed as following (SánchezLavega, 2011):

$$
n(r)=C r^{\left(1-3 \sigma_{e f f}\right) / \sigma_{e f f}} e^{-r / r_{e f f} \sigma_{e f f}}
$$

Where $\mathrm{r}$ is the radius of the particle, $r_{\text {eff }}$ is the effective radius and $\sigma_{\text {eff }}$ is the effective variance and $C$ a normalization constant. The effective radius is the average radius weighted by its geometrical cross-section:

$$
r_{e f f}=\frac{\int_{r 1}^{r 2} r \pi r^{2} n(r) d r}{\int_{r 1}^{r 2} \pi r^{2} n(r) d r}
$$

However, we can also define the mean radius $\left(r_{\text {mean }}\right)$ as the arithmetic mean of the radii between $r_{1}$ and $r_{2}$, in terms of number of particles:

$$
r_{\text {mean }}=\frac{\int_{r 1}^{r 2} r n(r) d r}{\int_{r 1}^{r 2} n(r) d r}
$$

Thus the mean and effective radii can be substantially different, as it is possible to find many small particles that reduce the mean radius, but do not contribute significantly to the effective scattering. This digression will be of interest later on.

Finally, the lower particle layer corresponds to the cloud that is expected to be located at some 1.4 bar, putatively formed by ammonia ice (Pérez-Hoyos et al., 2016). The cloud 
324 is assumed to be located between $P_{5}=1.0$ bar and $P_{6}=1.4$ bar with the particle optical thickness $\left(\tau_{\text {cloud }}\right)$ as the only free parameter. As in the stratospheric haze, the particle specific density (particles/gram) is assumed to be constant with altitude. In this layer, the phase function is described again by a Mie phase function with wavelengthindependent refractive index $m_{r}=1.43$ and $m_{i}=10^{-3}$. The particle size distribution is a log-normal distribution with an effective radius of $10 \mu \mathrm{m}$ and an effective variance of $0.1 \mu \mathrm{m}$ (West et al., 2009). As our a priori assumption we have taken the value of $\tau_{\text {cloud }}$ $=10 \pm 10$ from Pérez-Hoyos et al. (2016), who found $\tau_{\text {cloud }}>10$.

We show in Table 2 the set of 11 free parameters that define our inversion problem. To avoid over-fitting the imaginary refractive index, we started fitting the seven selected regions only at MT2, CB2 and MT3 filters, but fixing $m_{i}=10^{-3}$ for little tropospheric absorption at those wavelengths. Once an acceptable fit was found, we added shorter wavelength filters VIO and BL1, adding the refractive indices as free parameters. In this way we were able to find a maximum likelihood model that was acceptable in all the cases $\left(\chi^{2} / \mathrm{n}<1\right)$. We performed several initial runs to test if an increase of the number of free parameters at any of the layers (i.e. a more complex model) resulted in a better fit, with negative results.

\section{Modeling Results}

\subsection{General results}

We show in Fig. 5a, 5b and 5c the measured center-to-limb profiles compared to the best fit models for all regions and filters, and the different images (phase angles) used in this work. Table 3 summarizes the $\chi^{2} / n$ values for each region and filter, showing that fits are acceptable, but not always perfect. The mean $\chi^{2} / \mathrm{n}$ values are always smaller than 1 and most of the individual $\chi^{2} / \mathrm{n}$ values are less than 1 . However, the worst fit is found at MT2 filter in region 4, particularly at $\alpha=48^{\circ}$. Although in all regions the average $\chi^{2} / \mathrm{n}$ is less than 1, the worst global fit is found in region 4.

There are some causes that could partly explain the discrepancies between observations and models. First, the model could not be complex enough to account for the vertical distribution of tropospheric aerosols, hence affecting MT2 fitting, sensitive to pressures below the tropopause. Second, the assumption of sphericity is not likely to be correct for the tropospheric particles. Both aspects were already discussed in Pérez-Hoyos et al. 
355 (2016). A more complex model is not supported by the current data, and a better spectral resolution is required to increase its complexity. Regarding phase function, phase angle coverage is not enough to find influence due to variations in the scattering properties and it is mostly based on the dependence of optical thickness with wavelength, something that cannot be predicted independently with a synthetic phase function as the one used in Pérez-Hoyos et al. (2016). All in all, fits are within acceptable margins and therefore are taken as reliable estimations of the atmospheric parameters. Uncertainties and sensitivities will be discussed later on.

In order to rule out and quantify a possible systematic deviation of the limb-darkening in the different regions and filters and in order to improve our model we fitted both observations and best-fitting models to a Minnaert law (Pérez-Hoyos et al., 2005). (Fig. 6a and 6b). In this figure, we assume that all observations, at different phase angles, will follow the Minnaert law with the same Minnaert parameters, which seems to be the case. We do not find any systematic deviation in the limb darkening when compared with the original data, as the same filter or region can give differences as low as $2 \%$ or as high as $10 \%$, with average differences of $8 \%$. It must be noted that an alternate strategy could be used to take advantage of the better precision in the relative $(\sim 1 \%)$ than in the absolute photometry $(\sim 10 \%)$, by giving more weight to the limbdarkening than to the absolute nadir-corrected reflectivity value. While we did not perform a systematic study, our explorations of such strategy did not show any substantial improvement of the fits.

\subsection{Best-fitting results}

Table 4 shows the best-fitting values of the parameters showing that there is a clear latitudinal dependence for most of them.

The optical thickness of the stratospheric haze at $0.8 \mu \mathrm{m}$ ranges from $\tau_{s t r}=0.01 \pm 0.01$ (region 1) to $0.03 \pm 0.005$ (region 7). This is a change of a factor of 3 in the stratospheric particle density from the lower to the upper latitudes. Values at $0.4 \mu \mathrm{m}$ are also given, and found to be somewhat higher: $\tau_{s t r}=0.07 \pm 0.02$ (region 1) to $0.2 \pm 0.03$ (region 7), as it is expected for the sub-micron sized particles used here. These results are similar to those found in other studies, e.g. Perez-Hoyos et al. (2016). 
385

386

387

388

389

390

391

392

393

394

395

396

397

398

399

400

401

402

403

404

405

406

407

408

409

410

411

412

413

414

415

416

Regarding the parameters that describe the vertical distribution of the tropospheric haze, we give both the altitude (with respect to the 1 bar level) and the pressure level of the base of the haze. While the former is the actual free parameter in our model, the latter is easier to compare with previous results. There is a clear change from region 1 to region 7, as demonstrated by Figure 7. In the Hexagon Wave (region 4), the altitude of the base of the tropospheric haze reaches its lowest value before entering into the Polar Region (900 \pm 200 mbar.). Within the Hexagon and approaching the North Pole the value is $800 \pm 200$ mbar until reaching $1000 \pm 300$ mbar in the polar vortex. This is consistent with the dynamical interpretation that the North polar vortex is a region of subsidence with fewer particles and deeper clouds (Sánchez-Lavega et al., 2006). While some changes can be within the uncertainties of the parameters, there is an obvious difference between the values of the pressure of the base within the Polar Region (regions 4 to 7) and those found outside (regions 1 to 3 ).

The behavior of the concentration of tropospheric particles is similar to that of tropospheric haze base pressure, as shown in Figure 8. It decreases from region 1 to 3 ( $20 \pm 2$ to $15 \pm 1$ particles $/ \mathrm{cm}^{3}$ ) reaching a minimum value again in the region of the Hexagonal Wave (region 4, $4 \pm 0.5$ particles $/ \mathrm{cm}^{3}$ ). It slightly increases within the polar region (regions 5 and $6,5 \pm 0.5$ particles $/ \mathrm{cm}^{3}$ ), reaching a minimum value in the polar vortex of $2 \pm 0.5$ particles $/ \mathrm{cm}^{3}$. All these values, except the one at the most northerly latitude, are in good agreement with those found in other works such as Pérez-Hoyos et al. (2016).

In contrast to the concentration, the scale height of the tropospheric haze increases as we approach the North Pole from $18 \pm 0.1 \mathrm{~km}$ in region 1 to $50 \pm 0.1 \mathrm{~km}$ in region 7 . The scale height reaches the largest value beyond the Hexagon, where the values for region 6 (cloudy region at continuum wavelengths) are $32 \pm 2 \mathrm{~km}$ and $40 \pm 2 \mathrm{~km}$ for region 5 (region without cloud structure). These results range from half to one Saturnian gas scale height at the tropopause level, which is significantly higher than the values reported by Pérez-Hoyos et al. (2016) of around $\sim 0.2$ gas scale heights, or $7 \mathrm{~km}$.

The parameters in the preceding paragraphs can be integrated vertically (taking into account the particle cross-section) to provide the optical thickness $\tau_{\text {trop }}$ of the tropospheric haze. While this is not the actual parameter of our model, it is always useful for comparison with previous works. Outside the Polar Region we found (at 
$4170.8 \mu \mathrm{m}$ ) a maximum $\tau_{\text {trop }}=6 \pm 3$ (decreasing slightly until reaching the region of the 418 Hexagon Wave with $\tau_{\text {trop }}=1 \pm 0.5$ ). In the interior regions $\tau_{\text {trop }}=2 \pm 1$ for region 5, reaching the minimum value in the region 6 and within the polar vortex with $\tau_{\text {trop }}=$ $1 \pm 0.5$.These are low values and difficult to compare with the results of previous models since a high inclination orbit is required to observe more northerly latitudes, and they do not consider this type of observations, at least within the plane parallel approximation used here.

The particle size distribution also displays a dramatic dichotomy on both sides of the Hexagon Wave. For regions 1, 2 and 3, the values for $r_{\text {eff }}=7 \pm 0.1 \mu \mathrm{m}$ and $\sigma_{\text {eff }}=0.6 \pm 0.1$ $\mu \mathrm{m}$. This is quite a peculiar size distribution, as shown in Figure 9. It implies that the number of small particles is substantially higher than the larger ones. Since these have larger cross sections, they are the most effective ones scattering radiation, but the mean radius in terms of number of particles (using as a lower limit a reasonable value at molecular scales) is much smaller $r_{\text {mean }}=0.2 \pm 0.01 \mu \mathrm{m}$. However, when we reach region 4 we found again a distribution of particles centered around a mean value with values of $r_{\text {eff }}$ and $\sigma_{\text {eff }}$ respectively $2 \pm 0.2 \mu \mathrm{m}$ and $0.2 \pm 0.02 \mu \mathrm{m}$. In this case, the mean radius by number of particles is $1 \pm 0.01 \mu \mathrm{m}$. In region 5 the values of $r_{\text {eff }}$ and $\sigma_{\text {eff }}$ are $6 \pm 0.2$ and $0.03 \pm 0.02 \mu \mathrm{m}$, while in the region 6 they are respectively $4 \pm 0.4 \mu \mathrm{m}$ and $0.1 \pm 0.02 \mu \mathrm{m}$. Thus, the value of the mean radius is $5.0 \pm 0.5 \mu \mathrm{m}$ and $3 \pm 0.3 \mu \mathrm{m}$ for region 5 and region 6 . For the polar vortex the value of $r_{\text {eff }}$ and $\sigma_{\text {eff }}$ are respectively $4 \pm 0.1 \mu \mathrm{m}$ and $0.02 \pm 0.01$ $\mu \mathrm{m}$, while the mean radius is $4 \pm 0.4 \mu \mathrm{m}$. Even though particle size cannot be estimated with full confidence without a better coverage of scattering angles (and other parameters might influence the result), there is an evident change in the particle microphysics on either side of the Hexagon Wave. The differences in size distribution leads to a different behavior of the tropospheric optical thickness with wavelength, being nearly flat in the polar region and increasing strongly towards shorter wavelengths in regions 1 to 3 .

443 Figure 10 shows the imaginary refractive indexes for the tropospheric haze. For all 444 regions, the value of $m_{i}$ decreases with wavelength as we previously determined 445 (Sánchez-Lavega et al., 2006). We find again a difference between the values corresponding to the outer and the inner regions. In regions 1, 2 and 3 the values range from $(1 \pm 0.1) \times 10^{-4}$ (at $0.4 \mu \mathrm{m}$ ) to $(0.3 \pm 0.1) \times 10^{-7}$ (at $1 \mu \mathrm{m}$ ), whereas in regions 5, 6 and 7 the values range from $(0.2 \pm 0.04) \times 10^{-4}(0.4 \mu \mathrm{m})$ to $(0.1 \pm 0.07) \times 10^{-8}(1 \mu \mathrm{m})$. Region 4 again marks the difference, where the values are $(0.1 \pm 0.03) \times 10^{-2}$ for $0.4 \mu \mathrm{m}$ and 
$450(0.5 \pm 0.2) \times 10^{-6}$ for $1 \mu \mathrm{m}$. The imaginary refractive index strongly influences the particle

451

452

453

454

455

456

457

458

459

460

461

462

463

464

465

466

467

468

469

470

471

472

473

474

475

476

477

478

479

480

481

482 cross section, and these results may be degenerated with those for the particle size distribution, as they may also force an increase or decrease of particle cross-section.

We must highlight that the imaginary refractive indices a priori value was constant for all wavelengths. Motivated by the results found in the best-fit values of imaginary refractive indexes in regions 1,2 , and 3 , as well as the parameters of the particle size distribution, we have performed simulations in which the a priori values of imaginary refractive indexes were different for all wavelength $\left(10^{-2}(0.4 \mu \mathrm{m}), 10^{-3}(0.6 \mu \mathrm{m}), 10^{-4}(0.8\right.$ $\mu \mathrm{m})$ and $10^{-4}(1 \mu \mathrm{m})$ ); sub-optimal solutions were found that do not differ qualitatively from the results discussed in the preceding paragraphs. Finally, we do not have much sensitivity to the bottom cloud at the ammonia condensation levels, as the results were found to be above 10 for all cases, but too close to priors to be informative.

\subsection{Sensitivity to the Model Parameters.}

The process of finding the best fit starts from an a priori assumption of the free parameters, accompanied by their corresponding a priori error. Therefore, we assume some initial uncertainty for the parameters we are trying to retrieve. When our model conforms to the observations, we get both the a posteriori maximum likelihood value of each parameter and its corresponding error. A very useful measure of the information gained after the retrieval of one parameter is the improvement factor (Irwin et al., 2015), which compares the a priori and a posteriori relative errors. A low value of the improvement factor implies no information gain over the free parameter with respect to the a priori uncertainty, while a high value implies a substantial reduction of our initial uncertainty.

In this way, we find that for the stratospheric haze and bottom cloud opacities that the improvement factors are very low (3\% and 2\% respectively). Given this low sensitivity, we tested to fix these parameters for a few cases and found essentially the same results for the rest of parameters. For the tropospheric haze, we find a significant improvement factor for the base altitude, peak concentration and scale height of 93\%, 95\% and 78\%, respectively. For the rest of the free parameters the improvement factors are smaller, such as for the effective radius, the effective deviation and the imaginary refractive indexes around $15 \%$ for all of them. Therefore, the role of these parameters can be accepted to be secondary and the retrieval less informative. 
483 To stress the importance of the most informative free parameters, we have compared the best fit obtained with two other models, $1-\sigma$ above and below the nominal result. Figure 11 shows this comparison applied to region 1 . We observe that the most affected filters are MT2 and MT3. Another view of the same problem is to evaluate the change of the reflectivity for small (1\%) variations of each free parameter, also shown in Fig. 11. This is intended to show the geometrical variation of the sensitivity, which can be seen to be different in some cases at the central meridian or at the limb or terminator of the planet. Some parameters affect more strongly limb-darkening than others, that have a flatter behavior with wavelength. In Figure $11 \mathrm{~d}$, we see that the value of $\Delta \mathrm{I} / \mathrm{F}$ is very small for the VIO, BL1 and CB2 filters. The variation of $\Delta \mathrm{I} / \mathrm{F}$ is important in the filters MT2 and MT3, in which we observe that the most influential parameter is the scale height. For most cases, an increase in the parameter results in an increase of limb-darkening at a given phase angle, except a change in the scale height, which strongly reduces limbdarkening at the methane absorption filters.

We have extensively tested the impact that the bottom cloud may have in other free parameters of the tropospheric haze above it, particulary in its bottom pressure. We have considered models with extreme values of cloud's optical thickness and we have not observed variations of the lower pressure of the tropospheric haze in the any region. We find that the bottom pressure of the tropospheric haze is relatively robust and independent from the bottom cloud parameters which are neutral to the fitting procedure.

504

505

\section{Discussion.}

506

We have found that the retrieved optical thickness of both the stratospheric haze and tropospheric haze depend strongly on the latitude in the different reflectivity regions of the subpolar and Northern polar region of Saturn. As a general rule we note that the pressure of the base of the tropospheric haze increases towards polar latitudes (from $600 \pm 200$ mbar to $1000 \pm 300$ mbar in the vortex. However, the model is consistent with a bottom cloud of fixed altitude in the whole region but with some variability in its optical depth. Lack of sensitivity, however, precludes investigating alternate scenarios for the bottom cloud. 
515 As already explained, NEMESIS uses a Henyey-Greenstein approximation to the Mie516 calculated phase function, which neatly averages out features from purely spherical 517 particles such as the 'rainbow' and back-scattering 'glory'. In particular, we used the 518 Mie theory to generate the phase function at 41 scattering angles and then fitted the 519 parameters $\mathrm{f}, \mathrm{g}_{1}$ and $\mathrm{g}_{2}$ of the combined Henyey-Greenstein function to this Mie520 calculated phase function using the Levenberg-Marquardt technique. While there are 521 more sophisticated methods to retain some optical features as the glory (Kattawar, 522 1975), we find this approximation good enough for our purposes. In any case, the actual 523 values of the Henyey-Greenstein parameters used are given below for comparison with 524 previous works.

525 In Figure 12 we can see the comparison of both phase functions for region 3 and region 5265 with those obtained by Tomasko and Doose (1984) and that of Pérez Hoyos et al. 527 (2016). The phase function of Tomasko and Doose corresponds to latitudes of the 528 southern hemisphere where the two asymmetry parameters $g_{1}$ and $g_{2}$ are respectively 0.6 529 and -0.3 while parameter $f$ accounts for the relative weighting between them is 0.77 . For 530 the phase function of Pérez Hoyos et al. (2016) we have used the values of $g_{1}=0.81, g_{2}$ $531=-0.31$ and $f=0.85$ corresponding to latitudes of the northern polar region. The values 532 of Henyey-Greenstein approximation to the Mie-calculated phase function in this work 533 are $g_{1}=0.84, g_{2}=-0.41$ and $f=0.89$ for the region 3 and $g_{1}=0.78, g_{2}=-0.54$ and $f=$ 5340.98 for the region 5. These values are substantial different from those obtained by 535 Tomasko and Doose (1984) but similar to those by Pérez-Hoyos et al. (2016). However, 536 while the former values refer to the southern equatorial zone of the planet, the latter ones are taken for a more geographically similar region.

The Hexagonal Wave separates the polar region into two broad areas according to the particle properties, one enclosing the pole and the other outside the pole (Figs. 6, 7 and

541 8). This result is in agreement with the ones found by West et al. (2015) using Cassini 542 ISS polarization images where structural differences are observed inside and outside the hexagon. Poleward of the Hexagonal Wave above latitude $\sim 75^{\circ} \mathrm{N}$ (regions 5, 6 and 7) we found a large anticyclonic region (region 5) with an embedded field of discrete "puffy" clouds typically with a size of 100 and 400 kilometers (region 6) (Antuñano et al., 2018). The radiative transfer model indicates that this "puffy" cloud field is

547 distinguished from the surrounding region by a larger optical depth at the upper cloud 548 level. Few differences are noted in the tropospheric haze between the two regions. This 
549 supports the idea that denser clouds forms the "puffy" region, each element being 550 convective in origin, similar to the convective clusters of the mesoscale cellular 551 convection (MCC) found on Earth and ascending by the internal heat flow (Antuñano et 552 al., 2018). The whole polar region (regions 5, 6 and 7) has an optically denser 553 stratospheric haze (in particular in the UV). The origin of this haze could be related to 554 the bombardment of the upper atmosphere by particles trapped by the magnetic field 555 (West et al., 2009).

556 The cyclonic north polar vortex (region 7) extends from the pole to latitude $88.5^{\circ}$, where 557 peak velocities reach 140-160 $\mathrm{ms}^{-1}$ (Antuñano et al., 2015, Sayanagi et al., 2017). The 558 MT2 and MT3 filters show the cyclone to have low brightness relative to the 559 surroundings, but turns bright in violet wavelengths, consistent with low particle density 560 in the tropospheric haze and deeper clouds (Sánchez-Lavega et al., 2006; Baines et al., 561 2009). This is in agreement with our results since we found a minimum optical 562 thickness and low concentration of particles $\left(2 \pm 0.5\right.$ particles $\left./ \mathrm{cm}^{3}\right)$ in the cyclone 563 tropospheric haze. This is consistent with the polar cyclone being a "hole" within the 564 upper cloud system, where descending motions occur (Sánchez-Lavega et al., 2006; 565 Dyudina et al., 2009).

566 Saturn's Hexagon Wave encloses a fast eastward jet centered at $75.8^{\circ} \mathrm{N}$ that reaches a 567 mean peak velocity of $104 \pm 15 \mathrm{~ms}^{-1}$ (Antuñano et al., 2015). Our analysis shows that in 568 the Hexagon Wave region and in the latitudes it encloses the effective radius of the 569 tropospheric particles and the number density is smaller that outside. This is 570 accompanied by a descent of the haze base. Dynamical conditions are probably 571 involved in this dichotomy, but they have not been yet identified.

572 Equatorward of the Hexagon Wave latitude the base of the tropospheric haze reaches 573 higher altitudes and the particle effective radius, optical depth and particle density 574 increases. Dynamically this region contains a broad westward jet and a double-peaked 575 eastward jet, with alternating cyclonic and anticyclonic vorticity domains (Figure 2). 576 The banding we observe in these regions (Figure 1) does not match exactly the east577 west jet system. The belt/zone aspect is due to changes in the particle properties of the 578 hazes, with no requirement for additional haze layers, according to our model.

579

580

\section{Conclusions}


581 In this work we have performed a photometric analysis and radiative transfer modeling

582

583

584

585

586

587

588

589

590

591

592

593

594

595

596

597

598

599

600

601

602

603

604

605

606

607

608

609

610

611

612

613 of the subpolar and North polar regions of Saturn using a set of Cassini ISS images spanning a spectral range from $\sim 400 \mathrm{~nm}$ to $890 \mathrm{~nm}$, including two methane absorption bands at 727 and $890 \mathrm{~nm}$. We retrieve the vertical distribution and properties of the upper cloud and hazes from latitudes $\sim 50^{\circ}$ to $90^{\circ} \mathrm{N}$. The following highlights are the most important conclusions of our work.

- In this range of latitudes we distinguish seven regions. These seven regions account for the overall spectral and geometrical variation of reflectivity of the North Polar Region of Saturn.

- The Hexagon Wave and embedded jet stream represents a boundary in the aerosol properties between the outer and inner regions (West et al., 2015), which are substantially different in various aspects of the tropospheric haze (in particular the particle number density $(15 \pm 1$ part/cm3 for region 3 to $4 \pm 0.5$ part/cm3 for region 5), the scale height $(22 \pm 0.1 \mathrm{~km}$ for region 3 to $40 \pm 2 \mathrm{~km}$ for region 5), the base height ( $13 \pm 2 \mathrm{~km}$ for region 3 to $10 \pm 4 \mathrm{~km}$ for region 5), and size distribution $\left(r_{\text {eff }}=7 \pm 0.1 \mu \mathrm{m}\right.$ and $\sigma_{\text {eff }}=0.7 \pm 0.1 \mu \mathrm{m}$ for region 3 to $r_{\text {eff }}=$ $6 \pm 0.2 \mu \mathrm{m}$ and $\sigma_{e f f}=0.03 \pm 0.02 \mu \mathrm{m}$ for region 5).

- Both the optical thickness of stratospheric haze and the optical thickness of tropospheric haze gradually change with latitude. While the optical thickness of the stratospheric haze increases with latitude $(0.01 \pm 0.01$ for region 1 to $0.03 \pm 0.005$ for region 7 at $0.8 \mu \mathrm{m}$ ) the opposite is true for the tropospheric haze ( $6 \pm 3$ for region 1 to $1 \pm 0.5$ for region 7 at $0.8 \mu \mathrm{m}$ ). The variation of the total optical thickness of the tropospheric haze with latitude is correlated with the base pressure of this haze, which increases with latitude, showing a lower haze $(600 \pm 200$ mbar at the lowest latitudes to $1000 \pm 300$ mbar in the pole). The scale height also changes, resulting in quite a different vertical distribution of particles on either sides of the Hexagon Wave $(18 \pm 0.1 \mathrm{~km}$ for region 1 to $50 \pm 0.1 \mathrm{~km}$ for region 7).

- The optical thickness of the tropospheric haze shows a greater variation in the regions outside the Hexagon Wave $(6 \pm 3$ for region 1 at $0.8 \mu \mathrm{m})$ than in the interior regions $(1 \pm 0.5$ for region 7 at $0.8 \mu \mathrm{m})$.

- Aerosol properties (particle size distribution and refractive indices) seem to change on either side of the Hexagon Wave, too. It is unclear whether or not this 
reveals real particle properties changes or results from the dependence of optical thickness with wavelength.

- The low values of the optical thickness and particle concentrations found in the region enclosed by the Hexagon Wave, together with the retrieved behavior of the tropospheric haze base height, suggests that in this regions subsidence takes place.

- The polar vortex can be considered to be a depressed region of the atmosphere with possibly associated subsidence as quantified by Sánchez-Lavega et al. (2006).

- The radiative transfer model agrees with the consideration that puffy clouds have a convective origin similar to the convective clusters of the mesoscale cellular convection (MCC) found on Earth, and ascending by the internal heat flow (Antuñano et al., 2018).

While this work represents one snapshot for the situation of Saturn's Northern Polar atmosphere, it must be highlighted that the final orbits of the Cassini spacecraft are providing invaluable data with unprecedented spatial resolution for this part of the planet. Data acquired during Cassini's Grand Finale will surely complement the analysis shown here and will help to increase our understanding of Saturn's atmosphere.

\section{Acknowledgments}

We gratefully acknowledge the work of the Cassini ISS team that made the data available. This work was supported by the Spanish project AYA2015-65041-P (MINECO/FEDER, UE), Grupos Gobierno Vasco IT-765-13 and by Universidad del País Vasco UPV/EHU through program UFI11/55. We wish to thank Dr. R. West and Dr. U. Dyudina for their comments and suggestions during the review of the manuscript. 
641

\begin{tabular}{|c|c|c|c|c|c|}
\hline Image ID & Date & Filter & $\mathrm{B}\left({ }^{\circ}\right)$ & $\mathrm{B}^{\prime}\left({ }^{\circ}\right)$ & $\alpha\left({ }^{\circ}\right)$ \\
\hline W1748990013 & $06 / 03 / 2013$ & BL1 & 18.73 & 14.75 & 5.49 \\
\hline W1748990135 & $06 / 03 / 2013$ & MT3 & 18.73 & 14.73 & 5.52 \\
\hline W1748990103 & $06 / 03 / 2013$ & MT2 & 18.73 & 14.73 & 5.51 \\
\hline W1748990051 & $06 / 03 / 2013$ & & & & \\
\hline W1748990152 & $06 / 036 / 2013$ & CB2 & 18.73 & 14.72 & 5.53 \\
\hline W1750945864 & $06 / 13 / 2013$ & BL1 & 18.97 & 47.29 & 36.25 \\
\hline W1750945881 & $6 / 13 / 2$ & & 18.97 & & 36.24 \\
\hline W1750945932 & 13 & MT2 & 18.97 & 47.27 & 36.21 \\
\hline W1750945956 & $06 / 13 / 2013$ & MT3 & 18.97 & 47.26 & 36.20 \\
\hline W175 & & $\mathrm{CB}$ & 18.97 & 47.25 & 36.19 \\
\hline W1750953248 & $06 / 13 / 2$ & & 18.97 & & 32.62 \\
\hline W1750953265 & $06 / 13 / 2013$ & VIO & 18.97 & 44.82 & 32.61 \\
\hline W1750953316 & & & 18.97 & 44.81 & 32.59 \\
\hline W1 & & & 3.97 & 44.80 & 32.58 \\
\hline W17 & & $\mathrm{CB}$ & 18.97 & 44.79 & 32.57 \\
\hline W1750959544 & $06 / 13$ & & 18.97 & 42.73 & 29.66 \\
\hline & & & & & 29.65 \\
\hline W17 & $06 / 1$ & MT2 & 18.97 & 42.71 & 29.63 \\
\hline W17509: & & & 18.97 & 42.70 & 29.62 \\
\hline W1750959653 & & & 18.97 & & 29.61 \\
\hline & & & 97 & 50. & 40.67 \\
\hline W1750937305 & $06 / 14$ & VIO & 18.97 & 50.09 & 40.66 \\
\hline W1750937356 & & & 18.97 & 50.08 & 40.64 \\
\hline & & & & & 40.62 \\
\hline & & CB2 & 18.97 & 50.06 & 40.61 \\
\hline W1750934464 & $06 / 24$ & L1 & 18.97 & 51.00 & 42.18 \\
\hline & & & & & 42.17 \\
\hline W17509 & $06 / 2$ & MT2 & 18.97 & 50.98 & 42.15 \\
\hline W1750934556 & $06 / 24$ & MT3 & 18.97 & 50.97 & 42.13 \\
\hline & & & 18.97 & 50.97 & 42.12 \\
\hline & & & & 58.79 & \\
\hline & $06 / 26$ & VIO & 18.96 & 58.78 & 61.24 \\
\hline W1750902674 & $06 / 26 / 2013$ & MT3 & 18.96 & 58.78 & 61.21 \\
\hline & & & & & 61.19 \\
\hline & & & & & 61.17 \\
\hline W1750923064 & $06 / 26 / 20$ & & 18.97 & 54.43 & 48.57 \\
\hline W1750923081 & $06 / 26 / 20$ & VIO & 18.97 & 54.43 & 48.56 \\
\hline & & & & 54.41 & 48.53 \\
\hline & & & 18.97 & 54.41 & 48.52 \\
\hline W1750923173 & $06 / 26 / 2013$ & CB2 & 18.97 & 54.40 & 48.50 \\
\hline
\end{tabular}

642

643 Table 1: Summary of the observations, see the text for an explanation of the symbols used.

644 


\begin{tabular}{|c|c|c|c|}
\hline Layer & Parameter & Type & A priori \\
\hline \multirow[t]{7}{*}{ StratosphericHaze } & $P_{1}$ & Fixed & $1 \mathrm{mbar}$ \\
\hline & $P_{2}$ & Fixed & 100 mbar \\
\hline & $\tau_{s t r}$ & Free & $0.03 \pm 0.02$ \\
\hline & $m_{r}$ & Fixed & 1.43 \\
\hline & $m_{i}$ & Fixed & $10^{-3}$ \\
\hline & $r_{\text {eff }}$ & Fixed & $0.15 \mu \mathrm{m}$ \\
\hline & $r_{e f f}$ & Fixed & $0.1 \mu \mathrm{m}$ \\
\hline \multirow[t]{10}{*}{ Tropospheric Haze } & $P_{b o t}$ & Free & $625 \pm 96$ mbar \\
\hline & $N$ & Free & $10 \pm 5 \mathrm{part} / \mathrm{cm}^{3}$ \\
\hline & $H$ & Free & $35 \pm 10 \mathrm{~km}$ \\
\hline & $r_{e f f}$ & Free & $1.5 \pm 0.5 \mu \mathrm{m}$ \\
\hline & $\sigma_{\text {eff }}$ & Free & $0.1 \pm 0.1 \mu \mathrm{m}$ \\
\hline & $m_{r}$ & Fixed & 1.43 \\
\hline & $m_{i}(0.4 \mu \mathrm{m})$ & Free & $10^{-3} \pm 10^{-3}$ \\
\hline & $m_{i}(0.6 \mu \mathrm{m})$ & Free & $10^{-3} \pm 10^{-3}$ \\
\hline & $m_{i}(0.8 \mu \mathrm{m})$ & Free & $10^{-3} \pm 10^{-3}$ \\
\hline & $m_{i}(0.8 \mu \mathrm{m})$ & Free & $10^{-3} \pm 10^{-3}$ \\
\hline \multirow[t]{7}{*}{ Bottom Cloud } & $P_{5}$ & Fixed & 1.0 bar \\
\hline & $P_{6}$ & Fixed & 1.4 bar \\
\hline & $\tau_{\text {cloud }}$ & Free & $10 \pm 10$ \\
\hline & $m_{r}$ & Fixed & 1.43 \\
\hline & $m_{i}$ & Fixed & $10^{-3}$ \\
\hline & $r_{\text {eff }}$ & Fixed & $10 \mu \mathrm{m}$ \\
\hline & $\sigma_{e f f}$ & Fixed & $0.1 \mu \mathrm{m}$ \\
\hline
\end{tabular}

646

647 Table 2: Model atmosphere parameters. 
649

\begin{tabular}{|c|c|c|c|c|c|c|c|c|}
\hline Filter & Region 1 & Region 2 & Region 3 & Region4 & Region 5 & Region6 & Region 7 & $\begin{array}{c}\text { Average } \\
\text { filter }\end{array}$ \\
\hline VIO & 0.553 & 0.564 & 0.848 & 0.625 & 0.886 & 0.523 & 0.233 & 0.604 \\
\hline BL1 & 0.462 & 0.490 & 0.479 & 1.460 & 1.059 & 1.236 & 0.073 & 0.741 \\
\hline MT2 & 0.876 & 1.128 & 1.100 & 2.225 & 0.692 & 0.822 & 0.860 & 1.100 \\
\hline CB2 & 0.341 & 0.442 & 0.512 & 0.656 & 0.164 & 0.646 & 0.217 & 0.425 \\
\hline MT3 & 1.603 & 0.902 & 0.884 & 0.603 & 0.383 & 0.927 & 0.427 & 0.818 \\
\hline Region Average & 0.772 & 0.705 & 0.765 & 0.994 & 0.637 & 0.831 & 0.362 & \\
\hline
\end{tabular}

650

651 Table 3: $\chi^{2} / \mathrm{n}$ values for each region and filter.

652 


\begin{tabular}{|c|c|c|c|c|c|c|c|}
\hline & Region 1 & Region2 & Region 3 & Region 4 & Region 5 & Region 6 & Region 7 \\
\hline \multicolumn{8}{|l|}{$\begin{array}{c}\text { Stratospheric } \\
\text { Haze }\end{array}$} \\
\hline$\tau_{s t r}(0.8 \mu \mathrm{m})$ & $0.01 \pm 0.01$ & $0.03 \pm 0.01$ & $0.01 \pm 0.05$ & $0.01 \pm 0.001$ & $0.03 \pm 0.006$ & $0.03 \pm 0.005$ & $0.03 \pm 0.005$ \\
\hline$\tau_{s t r}(0.4 \mu \mathrm{m})$ & $0.07 \pm 0.02$ & $0.2 \pm 0.04$ & $0.08 \pm 0.02$ & $0.06 \pm 0.01$ & $0.2 \pm 0.04$ & $0.2 \pm 0.03$ & $0.2 \pm 0.03$ \\
\hline \multicolumn{8}{|c|}{ TroposphericHaze } \\
\hline$z(\mathrm{~km})$ & $30 \pm 6$ & $20 \pm 4$ & $13 \pm 2$ & $5 \pm 1$ & $10 \pm 4$ & $10 \pm 4$ & $0.2 \pm 0.1$ \\
\hline$P_{b o t}(\mathrm{mbar})$ & $600 \pm 200$ & $700 \pm 100$ & $800 \pm 100$ & $900 \pm 200$ & $800 \pm 200$ & $800 \pm 200$ & $1000 \pm 300$ \\
\hline$N($ part/cm 3$)$ & $20 \pm 2$ & $19 \pm 2$ & $15 \pm 1$ & $4 \pm 0.5$ & $4 \pm 0.5$ & $5 \pm 0.5$ & $2 \pm 0.5$ \\
\hline$H(\mathrm{~km})$ & $18 \pm 0.1$ & $20 \pm 0.1$ & $22 \pm 0.1$ & $30 \pm 0.1$ & $40 \pm 2$ & $32 \pm 2$ & $50 \pm 0.1$ \\
\hline$\tau_{\text {trop }}(0.8 \mu \mathrm{m})$ & $6 \pm 3$ & $5 \pm 2$ & $4 \pm 2$ & $1 \pm 0.5$ & $2 \pm 1$ & $1 \pm 0.5$ & $1 \pm 0.5$ \\
\hline$r_{e f f}(\mu \mathrm{m})$ & $7 \pm 0.1$ & $7 \pm 0.1$ & $7 \pm 0.1$ & $2 \pm 0.2$ & $6 \pm 0.2$ & $4 \pm 0.4$ & $4 \pm 0.10$ \\
\hline$\sigma_{e f f}(\mu \mathrm{m})$ & $0.6 \pm 0.10$ & $0.6 \pm 0.01$ & $0.7 \pm 0.1$ & $0.2 \pm 0.02$ & $0.03 \pm 0.02$ & $0.1 \pm 0.02$ & $0.02 \pm 0.01$ \\
\hline$r(\mu \mathrm{m})$ & $0.2 \pm 0.01$ & $0.1 \pm 0.01$ & $0.1 \pm 0.01$ & $1 \pm 0.01$ & $5 \pm 0.5$ & $3 \pm 0.3$ & $4 \pm 0.4$ \\
\hline$m_{i}(0.4 \mu \mathrm{m})$ & $1 \pm 0.1 \mathrm{E}-04$ & $0.7 \pm 0.1 \mathrm{E}-04$ & $0.1 \pm 0.01 \mathrm{E}-03$ & $0.1 \pm 0.03 \mathrm{E}-02$ & $0.2 \pm 0.04 \mathrm{E}-04$ & $0.2 \pm 0.1 \mathrm{E}-04$ & $0.2 \pm 0.001 \mathrm{E}-03$ \\
\hline$m_{i}(0.6 \mu \mathrm{m})$ & $0.7 \pm 0.2 \mathrm{E}-04$ & $0.6 \pm 0.2 \mathrm{E}-04$ & $0.7 \pm 0.2 \mathrm{E}-04$ & $0.3 \pm 0.05 \mathrm{E}-03$ & $0.4 \pm 0.2 \mathrm{E}-06$ & $0.6 \pm 0.5 \mathrm{E}-05$ & $0.4 \pm 0.01 \mathrm{E}-07$ \\
\hline$m_{i}(0.8 \mu \mathrm{m})$ & $0.3 \pm 0.2 \mathrm{E}-04$ & $0.4 \pm 0.1 \mathrm{E}-04$ & $0.5 \pm 0.2 \mathrm{E}-04$ & $1 \pm 0.001 \mathrm{E}-04$ & $0.8 \pm 0.1 \mathrm{E}-07$ & $0.6 \pm 0.2 \mathrm{E}-06$ & $0.8 \pm 0.02 \mathrm{E}-08$ \\
\hline$m_{i}(1.0 \mu \mathrm{m})$ & $0.3 \pm 0.1 \mathrm{E}-07$ & $0.4 \pm 0.1 \mathrm{E}-07$ & $0.5 \pm 0.1 \mathrm{E}-07$ & $0.5 \pm 0.2 \mathrm{E}-06$ & $0.1 \pm 0.07 \mathrm{E}-08$ & $0.5 \pm 0.5 \mathrm{E}-08$ & $0.1 \pm 0.03 \mathrm{E}-08$ \\
\hline \multicolumn{8}{|l|}{ Cloud } \\
\hline$\tau_{\text {cloud }}(0.8 \mu \mathrm{m})$ & $15 \pm 3$ & $24 \pm 5$ & $25 \pm 5$ & $11 \pm 2$ & $10 \pm 2$ & $16 \pm 9$ & $10 \pm 2$ \\
\hline
\end{tabular}

653

654 Table 4: Best-fit parameter values and their uncertainties.

655

656 


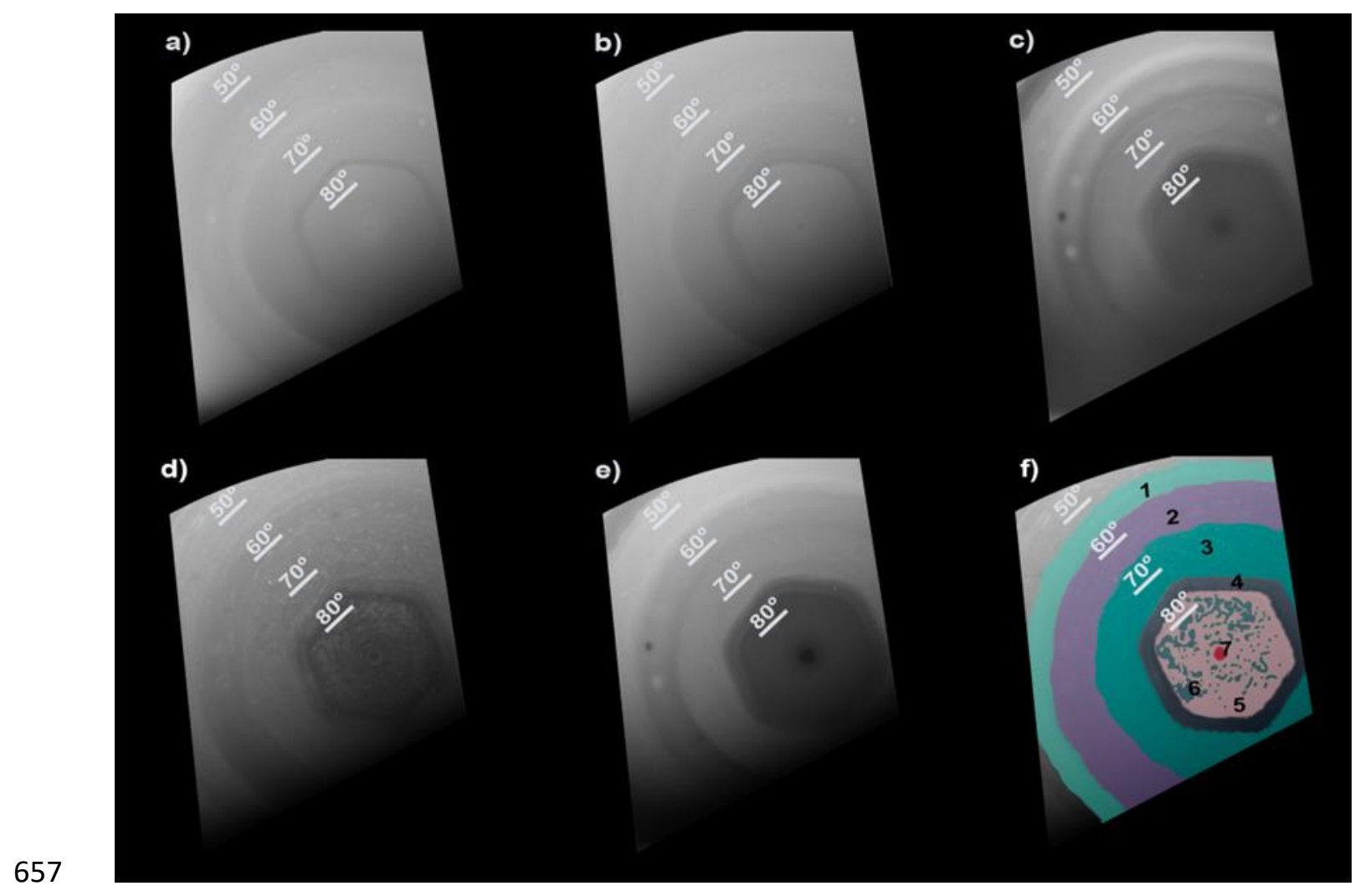

658 Figure 1: Polar projections of the images taken on June 26, 2013 for the five filters 659 used in this work: (a) VIO; (b) BL1; (c) MT3; (d) MT2; and (e) CB2. Panel (f) shows 660 the spatial distribution of the seven data clusters analyzed here.

661 


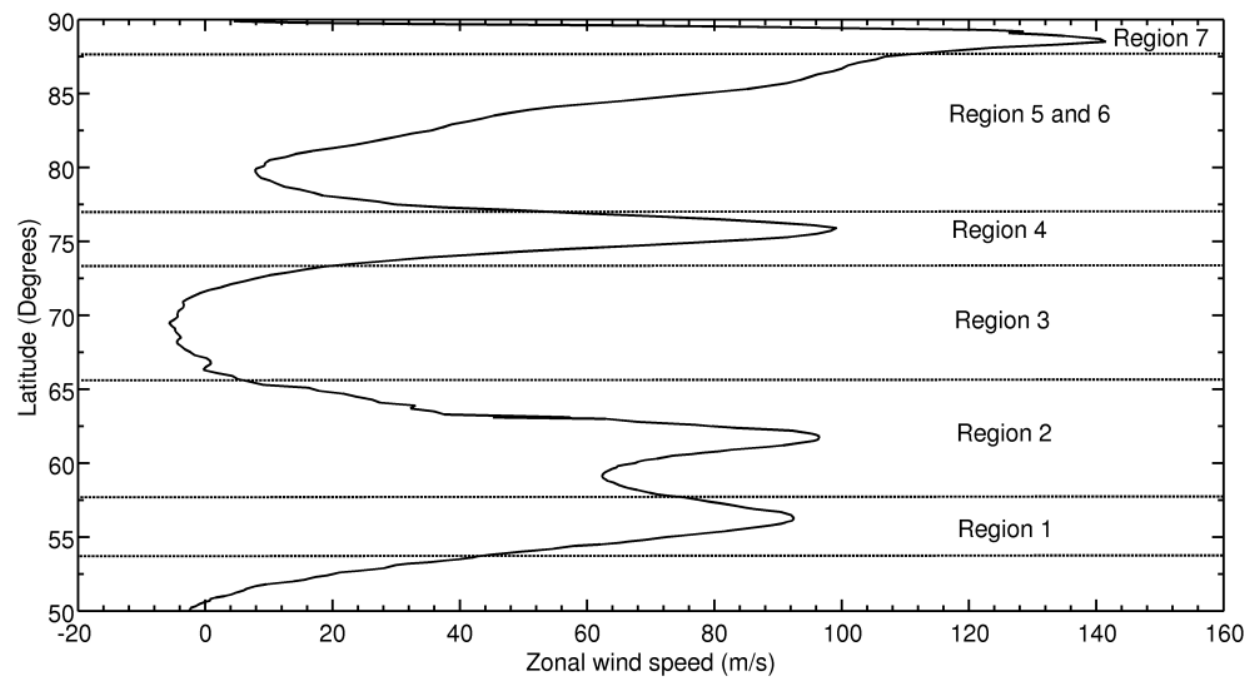

664 Figure 2: Zonal wind profiles for the seven regions identified in this work. The values were 665 obtained with Cassini ISS images with the CB2 filter in the years 2004, 2009 (Garcia-Melendo 666 et al., 2011) and 2013 (Antuñano et al., 2015). 


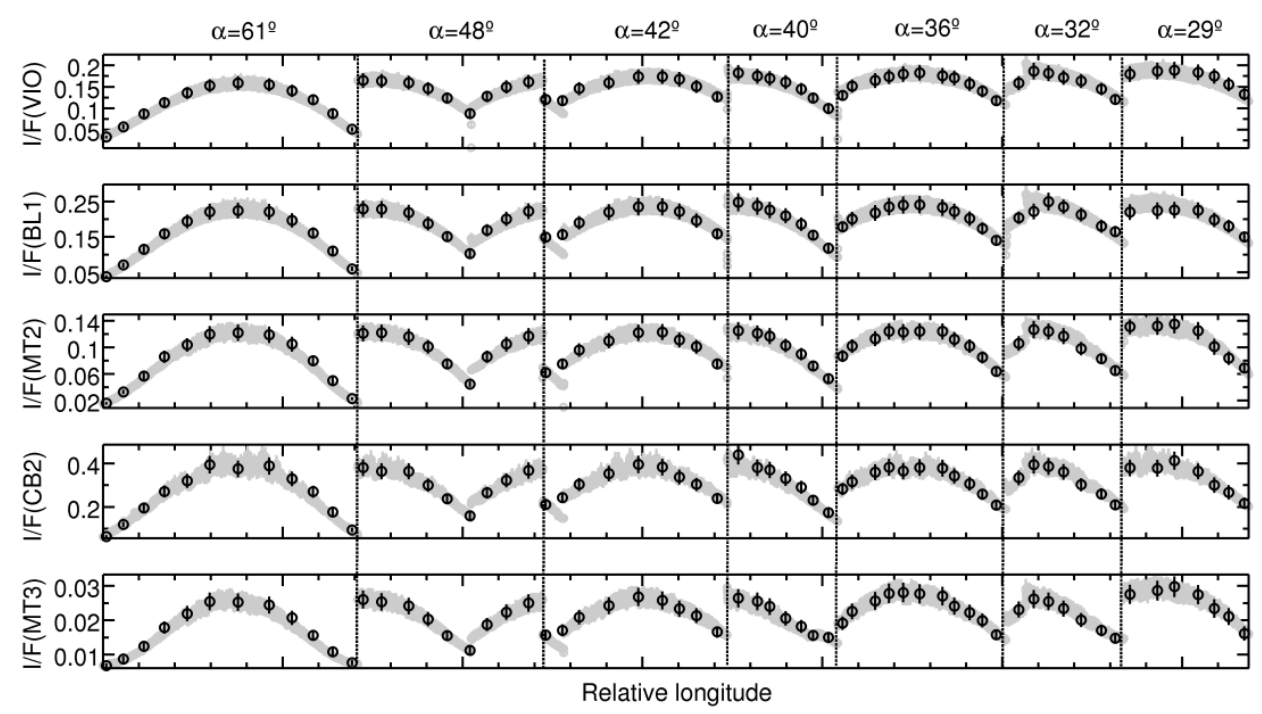

669 Figure 3: Reflectivity values for region $3\left(65^{\circ}\right.$ to $\left.73^{\circ} \mathrm{N}\right)$ for all filters are shown (grey line) 670 together with their average and standard deviation (black circles). The points shown here are 671 those used as inputs for the model. Vertical lines divide regions observed in every imaged, at 672 different phase angles $(\alpha)$. In this and some of the following figures, we use longitude measured 673 in degrees (every tick is $10^{\circ}$ ) from an arbitrary reference longitude. The changes in the 674 reflectivity with the longitude for each phase angle are due to the changes in the incidence and 675 emission angles. 

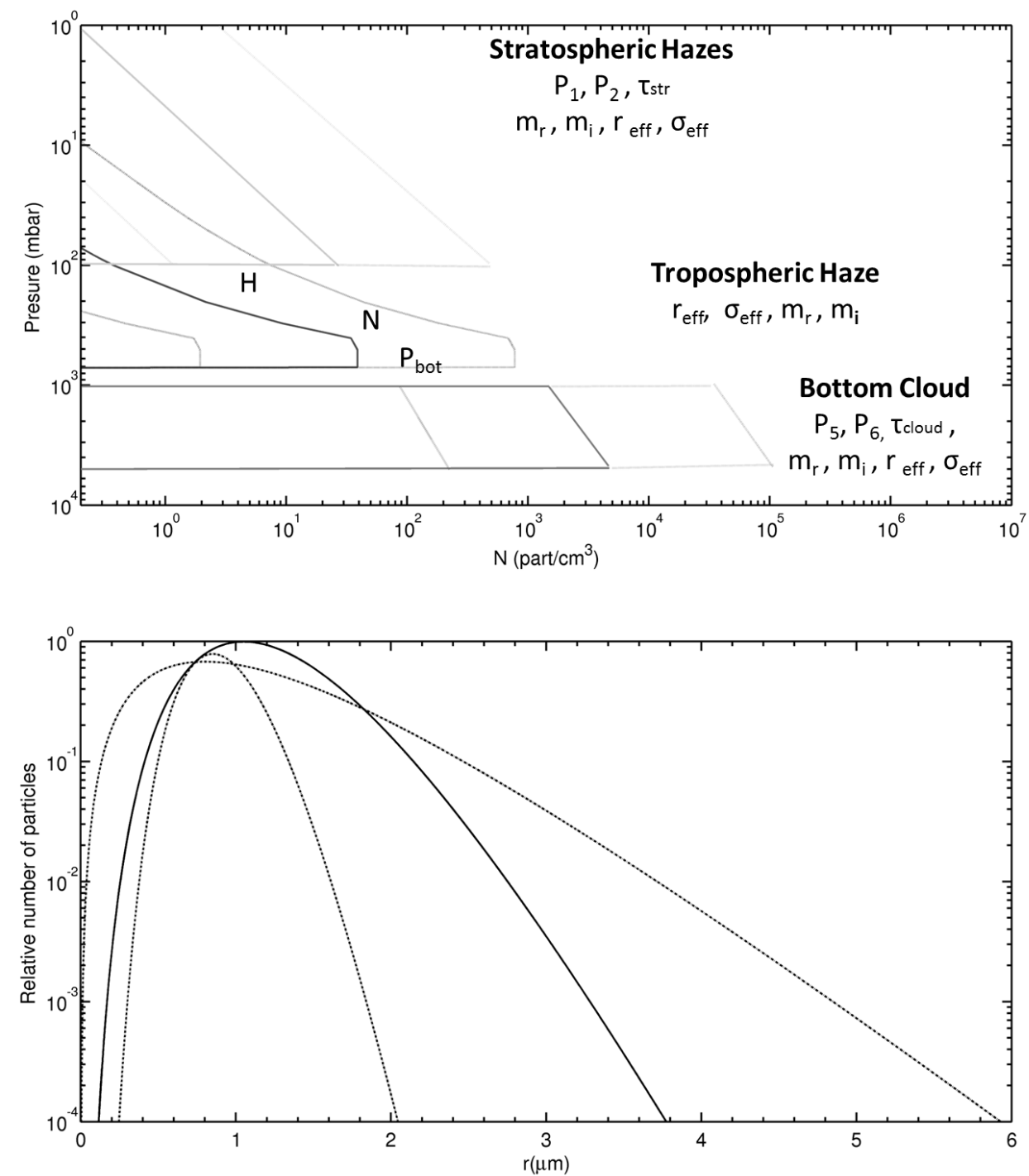

677 Figure 4: Top panel shows the a priori assumptions (solid lines) and their corresponding 678 uncertainties (gray lines) for the vertical distribution of particles. Parameters of the model for 679 each layer are also indicated (see the text for a full explanation). Bottom panel shows the a priori size distribution of tropospheric particles (solid line), and uncertainties (dashed lines). 


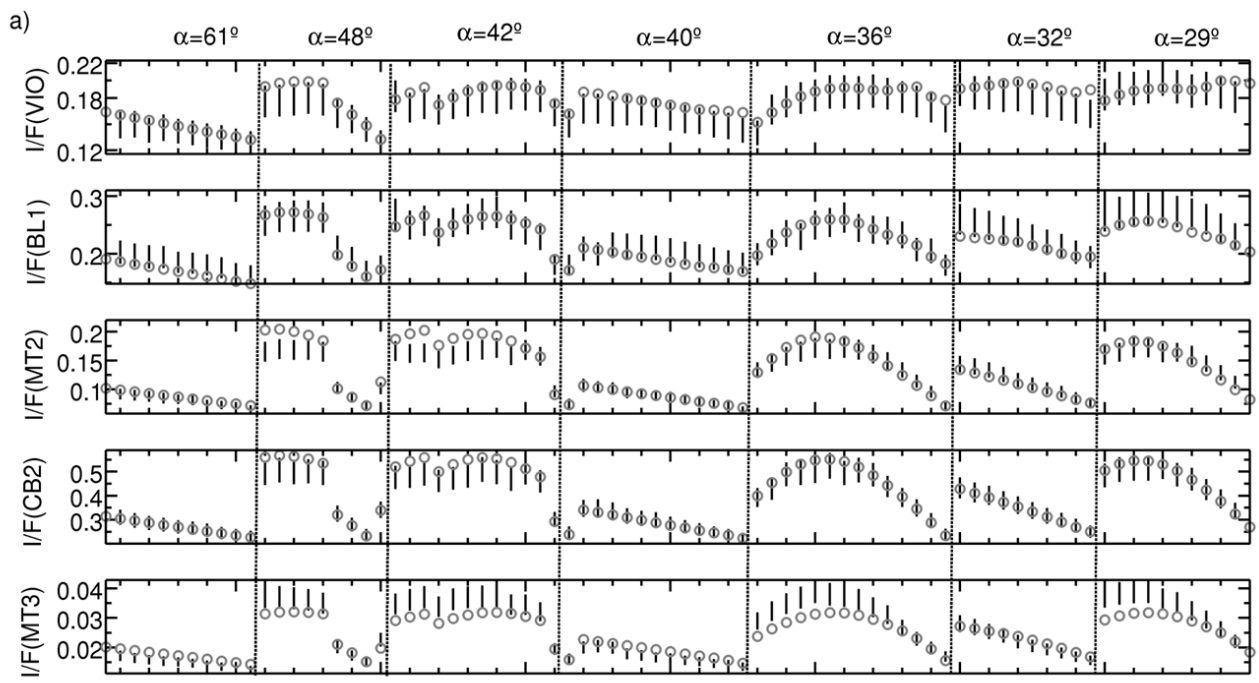

b)

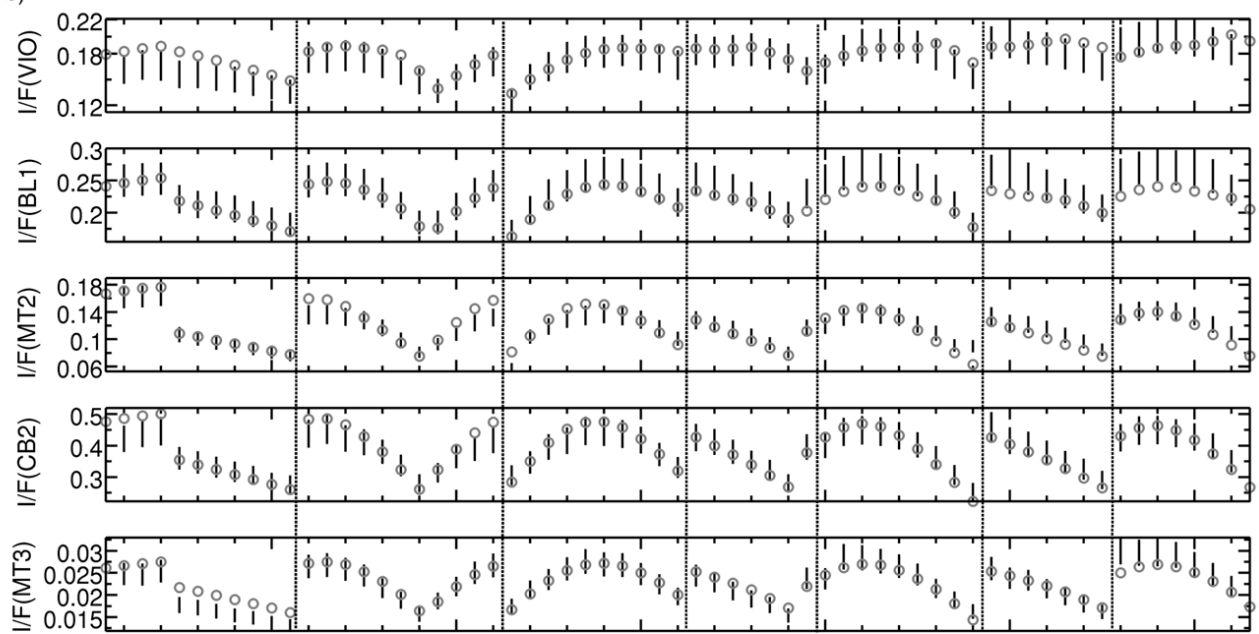
c)

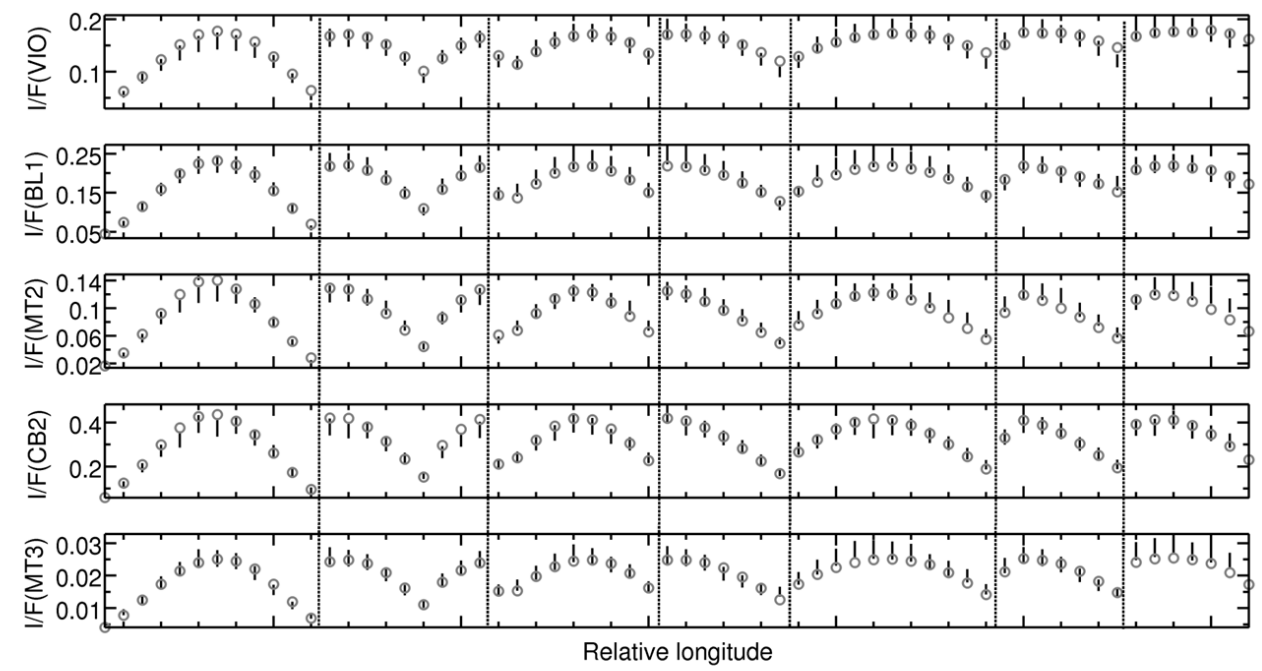


Figure 5a: Comparison of best fits models with observed values of reflectivity. Gray circles are used for the modeled values and the black lines correspond to the observed reflectivity and its corresponding error bar. For an explanation of $x$ axis, see Figure 3 caption. Panel a) shows Region 1, panel b) shows Region 2, and panel c) shows Region 3.

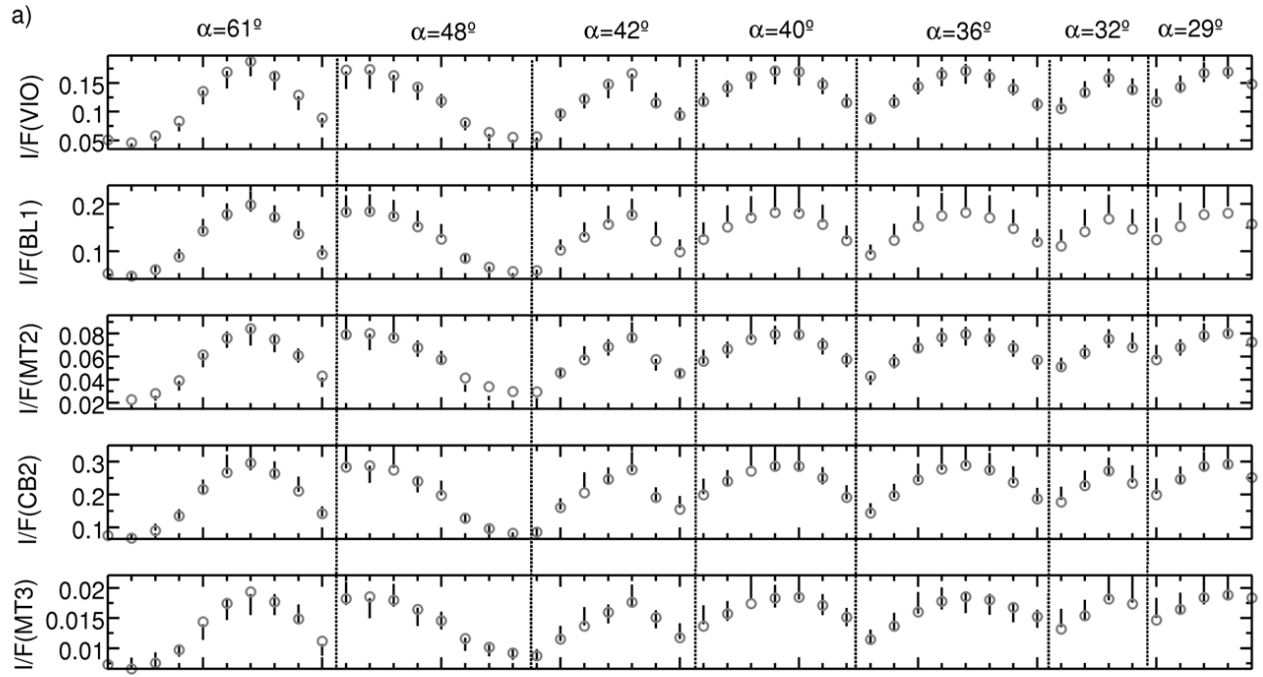

b)

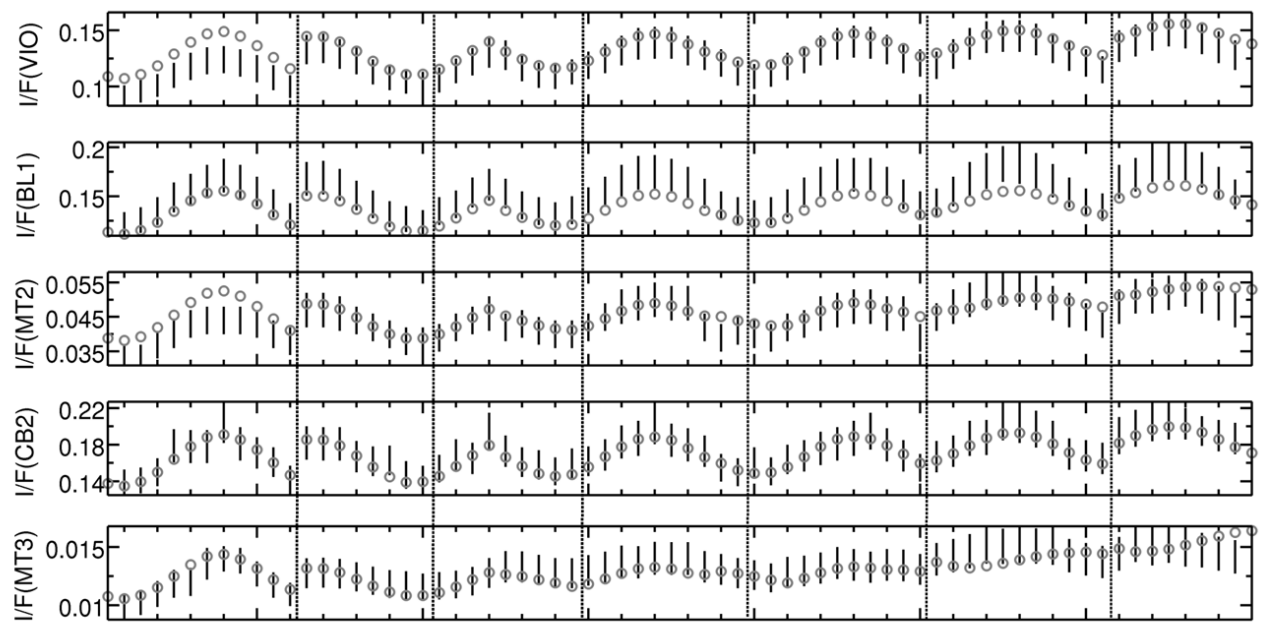

c)

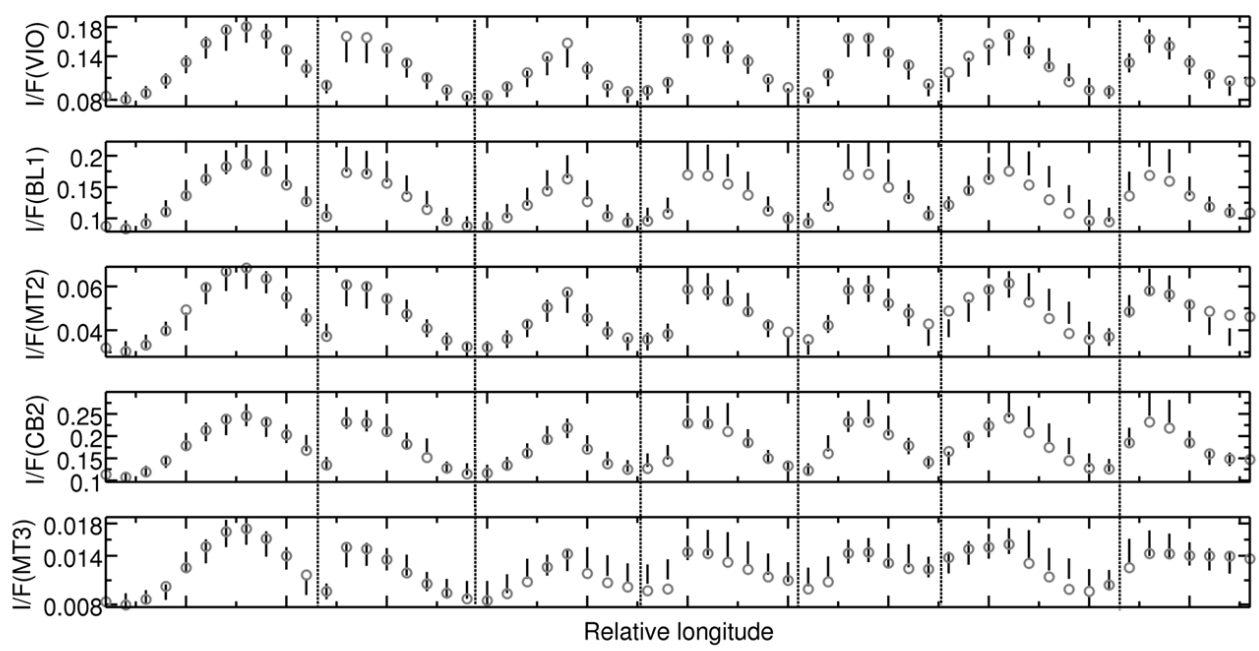


689 Figure 5b: Comparison of best fits models with observed values of reflectivity. Gray circles are 690 used for the modeled values and the black lines correspond to the observed reflectivity and its 691 corresponding error bar. Vertical dashed lines divide each region into the different phase angles

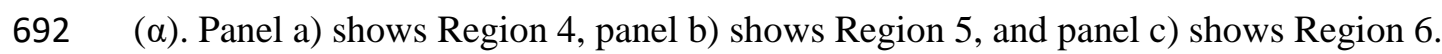

693 


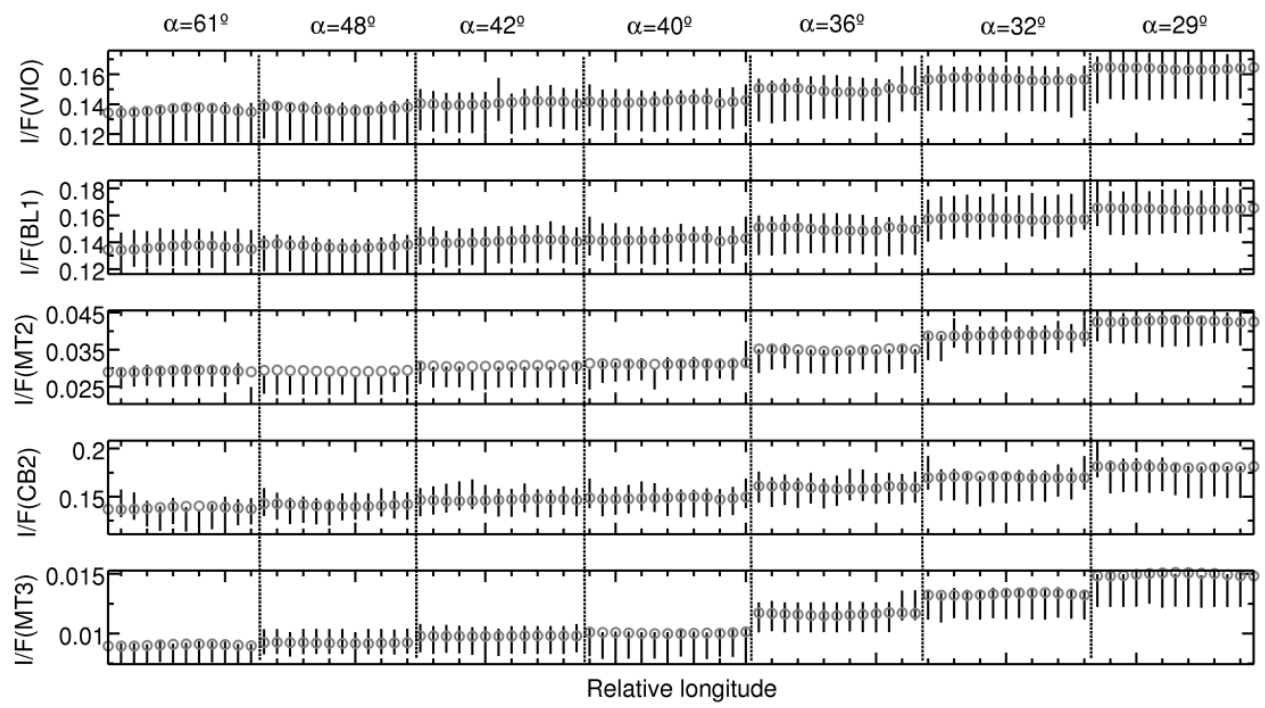

695

696 Figure 5c: Comparison of best fits models with observed values of reflectivity for region 7.

697 Gray circles are used for the modeled values and the black lines correspond to the observed 698 reflectivity and its corresponding error bar. For an explanation of $\mathrm{x}$ axis, see Figure 3 caption. 


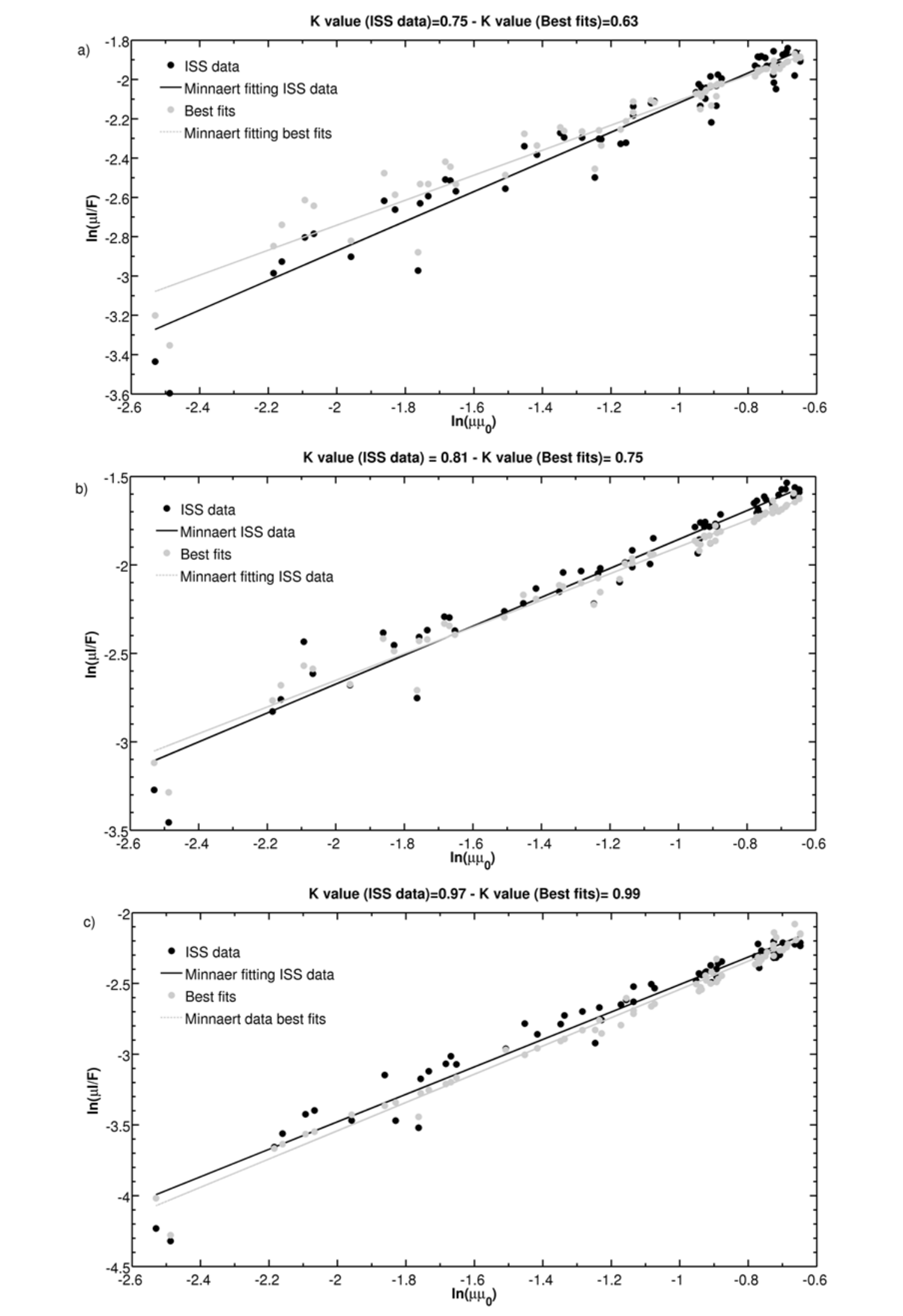

Figure 6a: Comparison of Minnaert limb-darkening model law of fitting ISS data with Minnaert limb-darkening model law of data best fits for region 3. Gray circles and lines are used for the modeled values and the black circles and lines correspond to the observed reflectivity. 
Panel a) shows VIO filter, panel b) shows BL1 filter, and panel c) shows MT2 filter. K is the Minnaert limb-darkening coefficient.

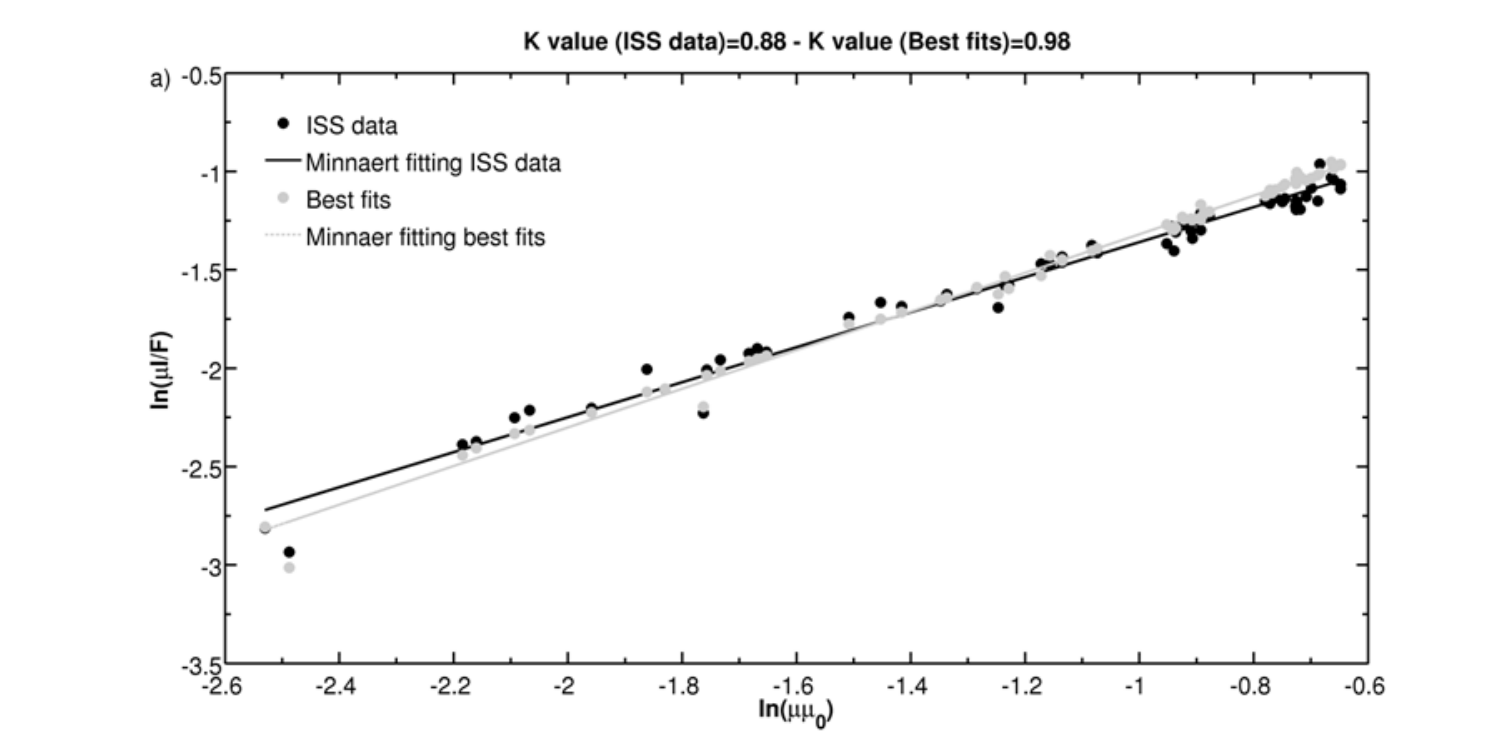

\section{7}

708 Figure 6b: Comparison of Minnaert limb-darkening model law of fitting ISS data with 709 Minnaert limb-darkening model law of data best fits for region 3. Gray circles and lines are used 710 for the modeled values and the black circles and lines correspond to the observed reflectivity. 711 Panel a) shows CB2 filter, panel b) shows MT3 filter. K is the Minnaert limb-darkening coefficient. 

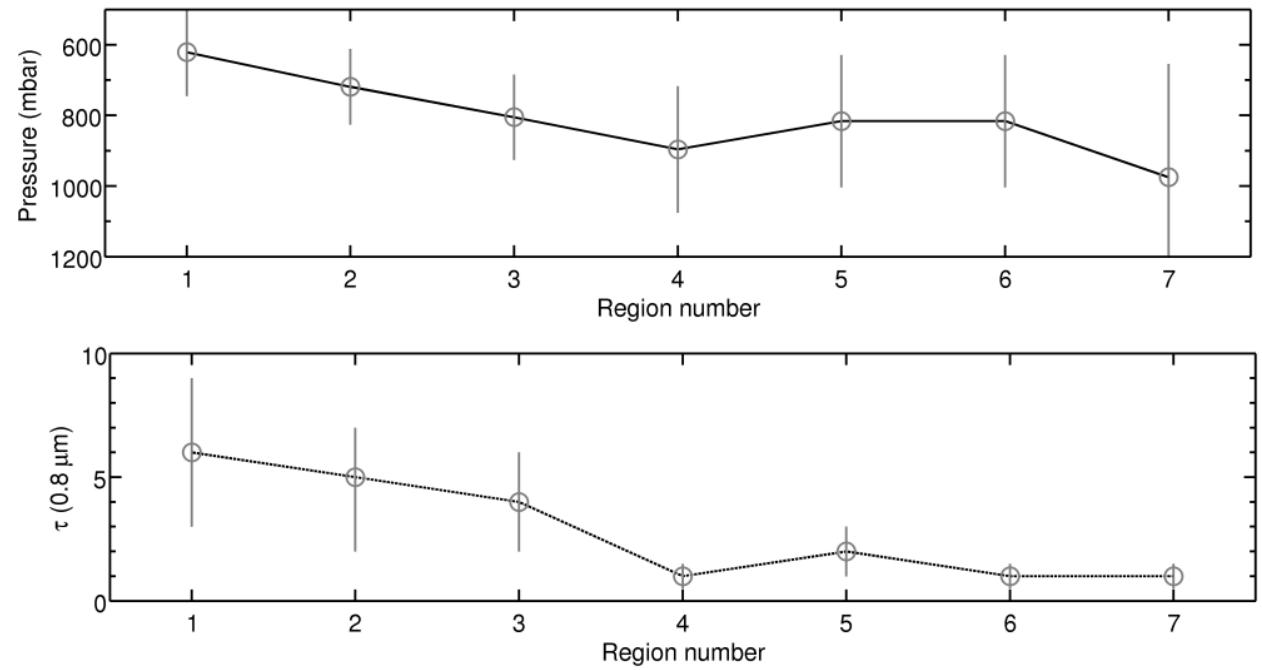

714

715 Figure 7: Altitude level for the base (in pressure) and optical thickness of the tropospheric haze 716 at $0.8 \mu \mathrm{m}$ as a function of the modeled regions, which roughly correspond to increasing latitude. 


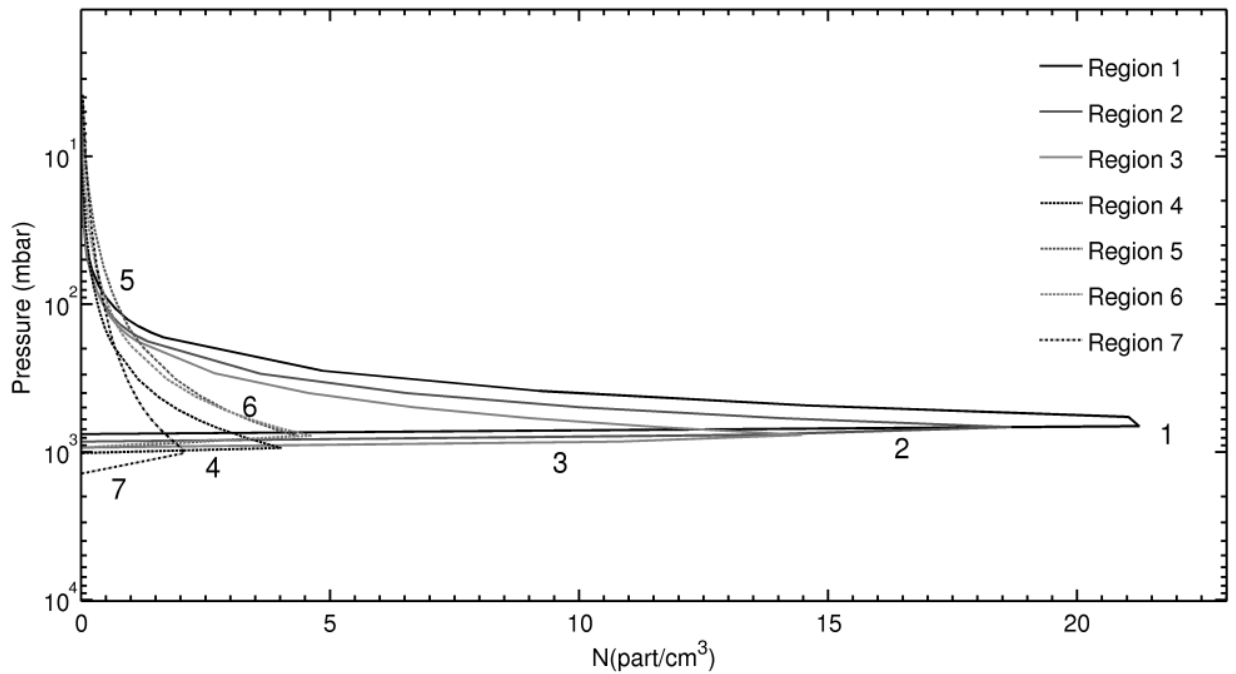

718

719 Figure 8: Retrieved particle number concentration as a function of pressure (or altitude) of the 720 tropospheric haze for all regions. Solid lines are used for regions equatorward of the Hexagon 721 Wave and dashed lines for the others. 


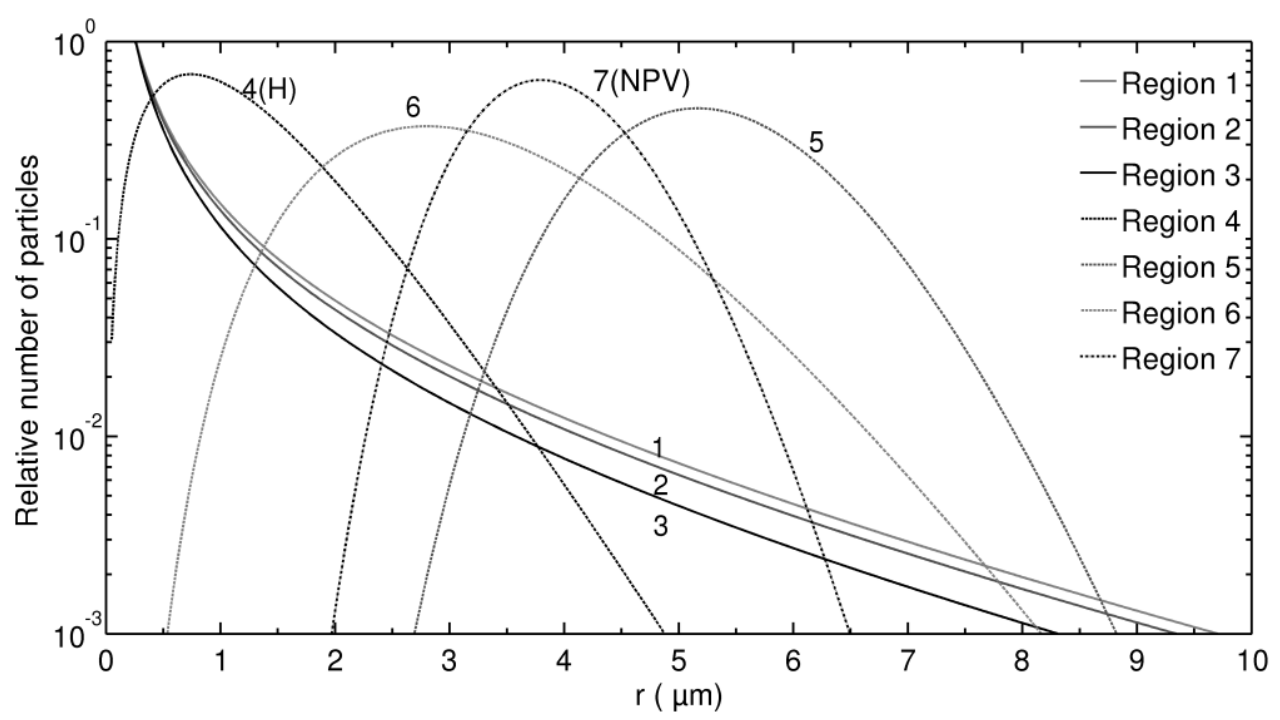

725 Figure 9: Size distributions of the tropospheric particles for all regions. Solid lines are used for 726 regions equatorward of the Hexagon Wave and dashed lines for the others. $\mathrm{H}$ stands for 727 Hexagon Wave and NPV for North Pole Vortex. 


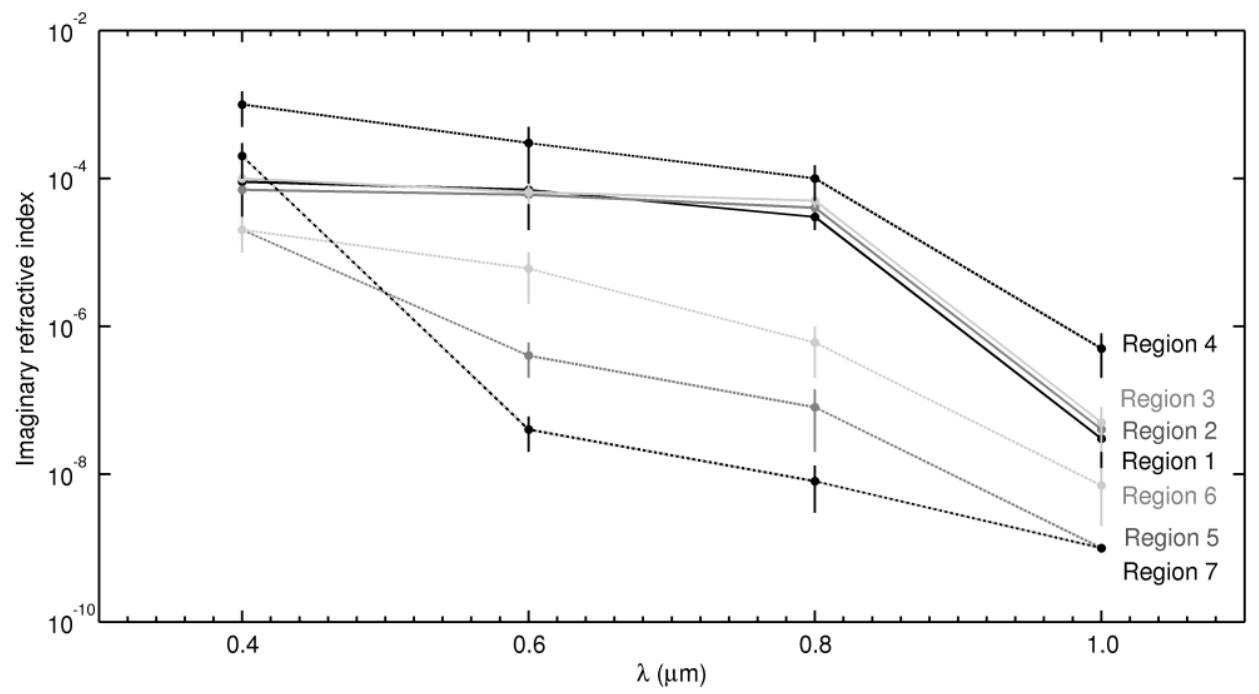

730 Figure 10: Retrieved imaginary refractive index of the tropospheric particles as a function of 731 wavelength for all regions. Solid lines are used for regions equatorward of the Hexagon Wave 732 and dashed lines for the others. 


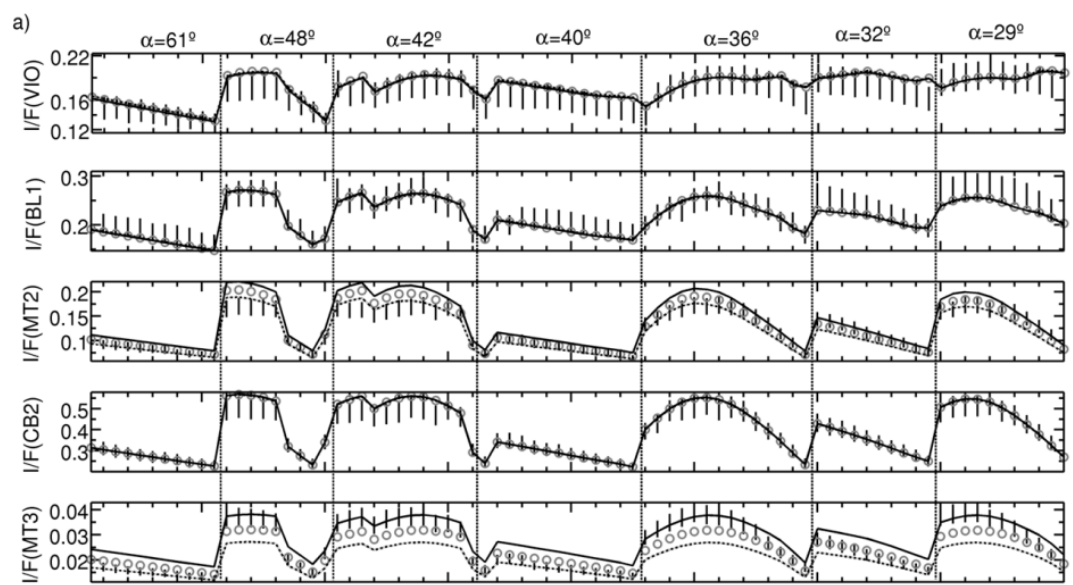

.

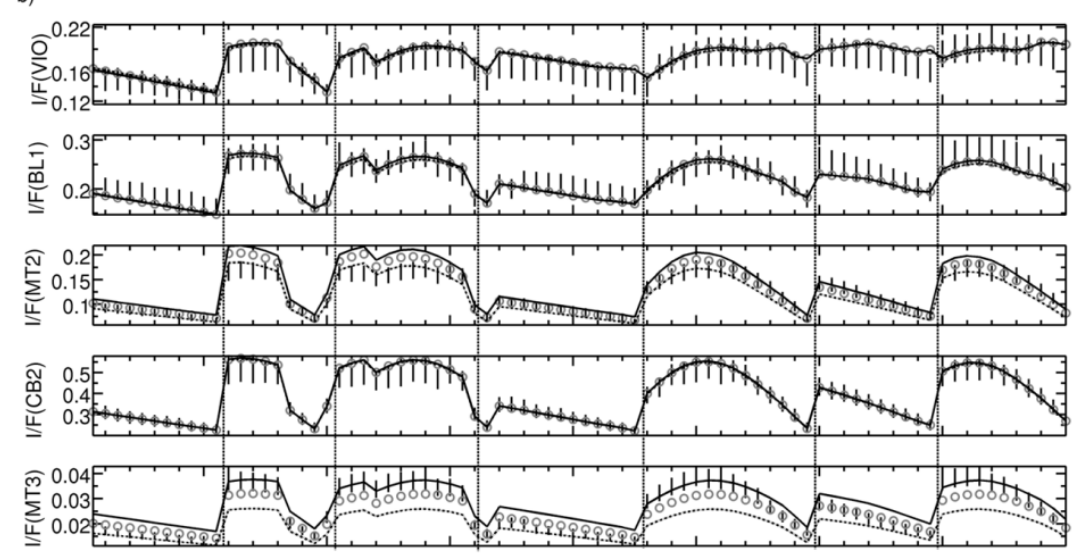

c)

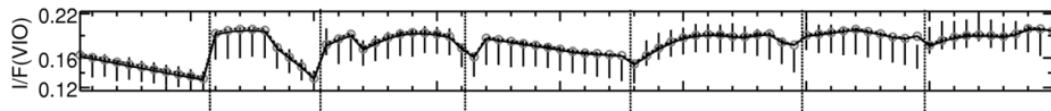

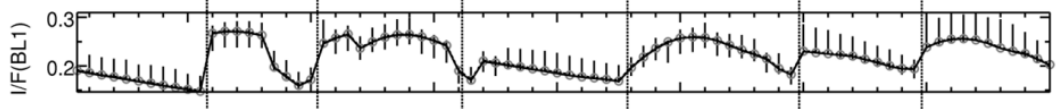

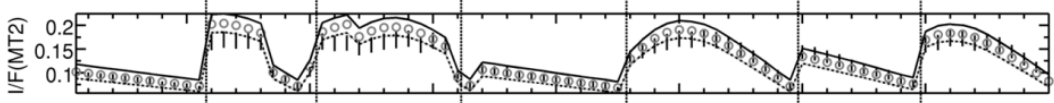

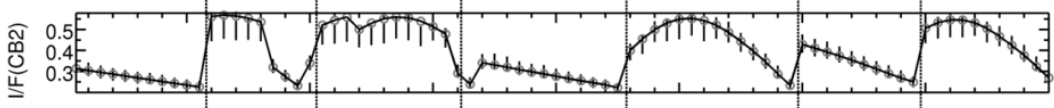

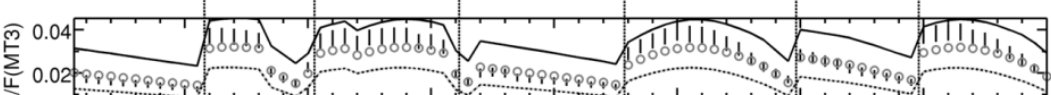

d)

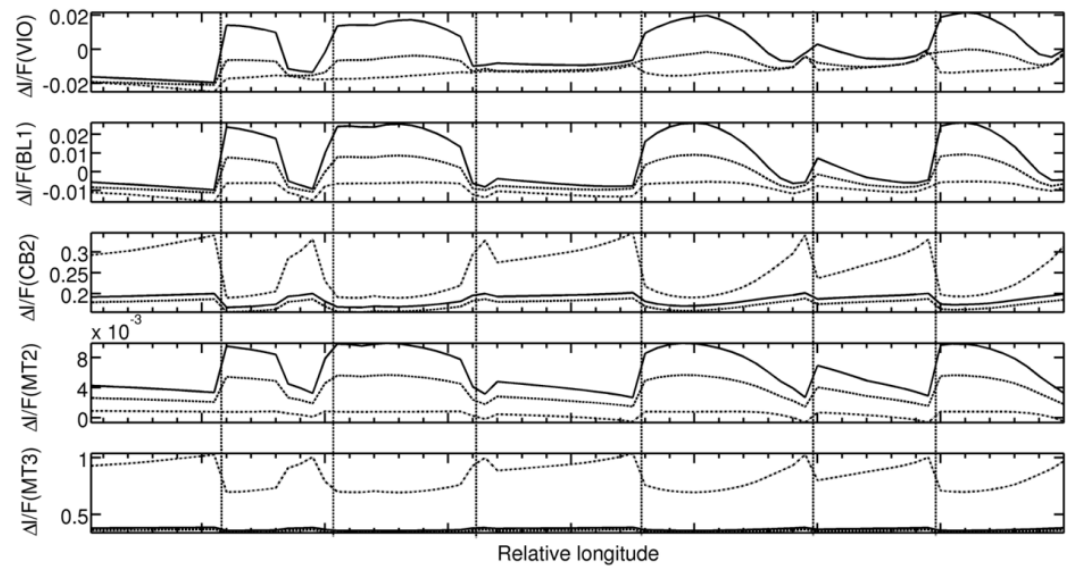


734 Figure 11: Sensitivity analysis based in a change for a given parameter of the tropospheric haze $7351-\sigma$ above (solid line) and below (dashed line) of the nominal value for the following 736 parameters: a) altitude of the haze base; b) particle peak concentration; c) scale height. In a), b) 737 and c) the gray circles correspond to the best model, the vertical solid lines are for the measured 738 values and their corresponding uncertainties. Panel shows the reflectivity change $\Delta \mathrm{V} / \mathrm{F}$ for small 739 changes $(1 \%)$ of the tropospheric parameters for each of the filters. Here, the solid line 740 corresponds to the variation of the height of the base of the tropospheric haze, the dashed line 741 corresponds to the variation of the particle peak concentration and the dot-dashed line the

742 variation of the scale height. Vertical lines separate the different phase angles $(\alpha)$. For an 743 explanation of $\mathrm{x}$ axis, see Figure 3 caption. 


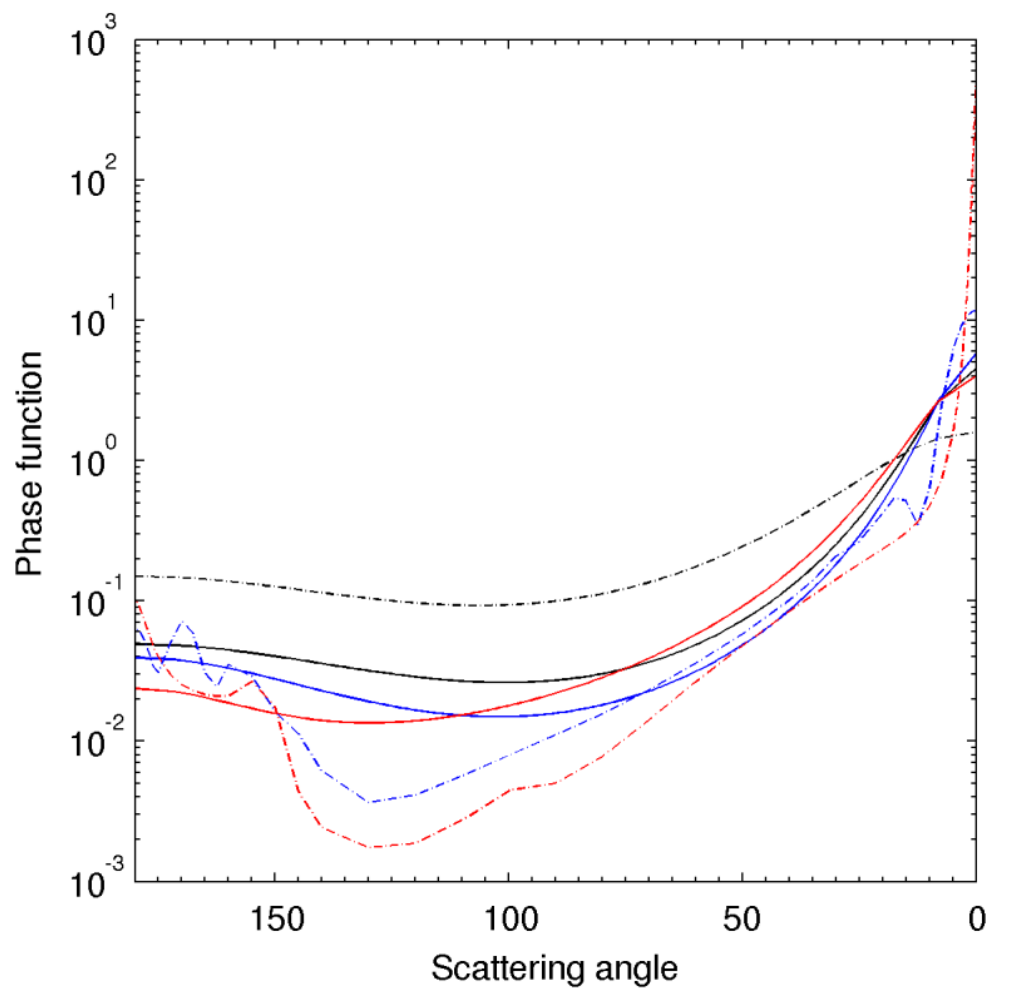

Figure 12. Henyey-Greenstein approximation to the Mie-calculated phase function and 748 comparison with the phase function of Tomasko and Doose and the phase function of Pérez749 Hoyos. The dashed black line corresponds to the phase function of Tomasko and doose (1984)). 750 The black line to the phase function of Pérez-Hoyos et al. 2016. The blue line is the 2HG of the 751 región 3 while the dashed blue line is the Mie phase function of that region. The red line corresponds to the $2 \mathrm{HG}$ of region 5 and the dashed red line to the Mie phase function of this region. 
755

756

757

758

759

760

761

762

763

764

765

766

767

768

769

770

771

772

773

774

775

776

777

778

779

780

781

782

783

784

785

786

787

788

789

790

791

792

\section{References}

Allison, M., D. A. Godfrey, and R. F. Beebe, 1990. A wave dynamical interpretation of Saturn's Polar Hexagon, Science, 247, 1061-1063.

Antuñano, A., Río-Gaztelurrutia, T., Sánchez-Lavega, A., 2015. Dynamics of Saturn's polar regions. Journal of Geophysical Research (Planets) 120(Feb.), 155-176.

Antuñano, A. Río-Gaztelurrutia, T., Sánchez-Lavega, A and Rodriguez_Aseguinolaza J., 2018. Cloud, morphology and dynamics in Saturn's Northern Polar Region. Icarus $299,117-132$.

Baines, K.H., Momary, T.W., Fletcher, L.N., et al., 2009. Saturn's north polar cyclone and hexagon at depth revealed by Cassini/VIMS.Planetary and Space Science 57 (Dec.), 1671-1681.

Barbosa-Aguiar, A. C., P. L. Read, Robin D. Wordsworth, T. Salter, and Y. H. Yamazaki, 2010, A laboratory model of Saturn's North Polar Hexagon, Icarus, 206, $755-763$.

Barrado-Izagirre, N., Sánchez-Lavega, A., Pérez-Hoyos, S., et al., 2008. Jupiter's polar clouds and waves from Cassini and HST images: 1993 - 2006. Icarus 194 (Mar.), 173185 .

Baines, K. H., Momary, T. W., Fletcher, L. N. et al., 2009. Saturn'snorth polar cyclone and hexagon at depth revealedby Cassini/VIMS. Planetary and Space Science, 57, 1671-1681.

Brown, R.H., Baines, K.H., Bellucci, G., et al., 2004. The Cassini Visual and Infrared Mapping Spectrometer (VIMS) Investigation. Space Science Reviews115 (Dec.), 111168.

Caldwell, J., T. Benoit, X.-M. Hua, C. D. Barnet, and J. A. Westphal, 1993. An observed drift of Saturn's Polar Spot by HST, Science, 260, 326-329.

del Río-Gaztelurrutia, T., Legarreta, J., Hueso, R., et al., 2010. A long-lived cyclone in Saturn's atmosphere: Observations and models. Icarus 209, 665-681.

Del Genio, A.D., R. K. Achterberg, K. H. Baines, F. M. Flasar, P.L. Read, A. SánchezLavega, A. P. Showman, 2009 . Saturn Atmospheric Structure and Dynamics. Chapter 6 in Saturn after Cassini-Huygens. M. Dougherty, L. Esposito and T. Krimigis (editors), Springer-Verlag, pp. 113-159.

Dyudina, U.A., Ingersoll, A.P., Ewald, S.P., Vasavada, A.R., West, R.A., Del Genio, A.D. , Barbara, J.M. , Porco, C.C. , Achterberg, R.K., Flasar, F.M. , Simon-Miller, A.A ., Fletcher, L.N., 2008. Dynamics of Saturn's south polar vortex. Science 319, 1801. 
793 Dyudina, U.A., et al., 2009. Saturn's south polar vortex compared to other large vortices 794 in the solar system, Icarus, 202, 240-248.

795 Fletcher, L.N., et al., 2008. Temperature and composition of Saturn's polar hot spots and Hexagon. Science 319, 79-81.

797 Fletcher, Leigh N., Irwin, P. G. J., Achterberg, R. K.; et al.,2016. Seasonal variability of 798 Saturn's tropospheric temperatures, winds and para- $\mathrm{H}-2$ from Cassini far-IR 799 spectroscopy. Icarus, 264, 137-159

800 Fouchet T., J. I. Moses, B.J. Conrath., 2009. Saturn: Composition and Chemistry. 801 Chapter 5 in Saturn after Cassini-Huygens. M. Dougherty, L. Esposito and T. Krimigis 802 (editors), Springer-Verlag, pp. 83-112.

803 García-Melendo, E., A. Sánchez-Lavega, J. F. Rojas, S. Pérez-Hoyos, and R. Hueso, 804 2009. Vertical shears in Saturn's eastward jets at cloud level. Icarus 201, 818-820.

805 García-Melendo, E., A. Sánchez-Lavega, S. Pérez-Hoyos, and R. Hueso, 2011. Saturn's 806 zonal wind profile in 2004 - 2009 from Cassini ISS images and its long-term variability. 807 Icarus $215,62-74$.

808 Godfrey, D.A., 1988. A hexagonal feature around Saturn's North Pole. Icarus 76, 335809356.

810 Hansen, J.E., Travis, L.D., 1974. Light scattering in planetary atmospheres. SpaceSci. 811 Rev. 16, 527-610.

812 Hueso, R., J. Legarreta, J. F. Rojas, J. Peralta, S. Pérez-Hoyo, T. del Río-Gaztelurrutia, 813 and A. Sánchez-Lavega, 2010.ThePlanetary Laboratory for Image Analysis (PLIA). 814 Adv. Space Res. 6, 1120-1138.

815 Irwin, P.G.J., Teanby, N.A., de Kok, R., et al., 2008. The NEMESIS planetary 816 atmosphere radiative transfer and retrieval tool. J. Quant. Spectrosc. Radiat. Transf. 109, $817 \quad 1136-1150$.

818 Irwin, P.G.J., Fletcher, L.N., Read, P.L., et al., 2015. Spectral analysis of Uranus' 2014 819 bright storm with VLT/SINFONI. Icarus 264, 72-89

820 Karkoschka, E., and M.G. Tomasko, 1993. Saturn's upper atmospheric hazes observed 821 by the Hubble Space Telescope. Icarus 126, 428-441.

822 Karkoschka, E., Tomasko, M.G., 2005. Saturn's vertical and latitudinal cloud structure 823 1991-2004 from HST imaging in 30 filters. Icarus 179, 195-221.

824 Kossin, J.P., and Schubert, W.H., 2001. Mesovortices, Polygonal Flow Patterns, and 825 Rapid Pressure Falls in Hurricane-Like Vortices. Journal of Atmospheric Sciences 826 58(Aug.), 2196-2209. 
828 Kattawar, G.W., 1975. A three parameters analytic phase function for multiple scattering calculations . J. Quant. Spect. \& Rad. Transfer 15, 839-849.

830 Lucarini, V., Saarinen, J.J., Peiponen, K.E., Vartiainen, E.M., 2005. Kramers831 KronigRelationsin Optical Materials Research.Springer-Verlag Berlin Heidelberg. 832 Germany.

MacQueen, J. B., 1967. Some methods for classication and analysis of multivariate observations. Proceedings of the Fifth Symposium on Math, Statistics, and Probability (pp. 281-297). Berkeley, CA: University of California Press.

Martonchik, J.V., Orton, G.S., Appleby, J.F., 1984. Optical properties of $\mathrm{NH}_{3}$ ice from the far infrared to the near ultraviolet. Appl. Optics 23, 541-547.

Morales-Juberías, R., K.M. Sayanagi, T.E. Dowling, and A.P. Ingersoll, 2011. Emergence of polar-jet polygons from jet instabilities in a Saturn model. Icarus 211, 1284-1293.

Ortiz, J.L, Moreno, M., And A. Molina., 1996. Saturn 1991-1993: Clouds and Hazes. Icarus 119, 53-66.

Pérez-Hoyos, S., Sánchez-Lavega, A., French, R.G., Rojas, J.F., 2005. Saturn's cloud structure and temporal evolution from ten years of Hubble space telescope images (1994-2003). Icarus 176, 155-174.

Pérez-Hoyos, S., J.F. Sanz-Requena, A. Sánchez-Lavega, M.H. Wong, H.B. Hammel, G.S. Orton, I. de Pater, A.A. Simon-Miller, J.T. Clarke, and K. Noll, 2012. Vertical cloud structure of the 2009 Jupiter impact base fromHST/WFC3 observations.Icarus $221,106-1078$.

Pérez-Hoyos S., José Francisco Sanz-Requena, Agustín Sánchez-Lavega, Patrick G.J. Irwin, Andrew Smithd, 2016. Saturn's tropospheric particles phase function and spatial distribution from Cassini ISS 2010-11 observations. Icarus 277, 1-18.

Porco, C.C., West, R.A., Squyres, S., et al., 2004. Cassini Imaging Science: Instrument Characteristics and Anticipated Scientific Investigations at Saturn. Space Science Reviews 115 (Dec.), 363-497.

Roman, M.T., Banfield, D., Gierasch, P.J., 2013. Saturn's cloud structure from Cassini ISS, Icarus 225. 93-110.

Sanz-Requena, J.F., Pérez-Hoyos, S., Sánchez-Lavega, A., et al., 2012. Cloud structure of Saturn's 2010 storm from ground-based visual imaging. Icarus 219, 142-149.

Sánchez-Lavega, A., J. Lecacheux, F. Colas, and P. Laques, 1993. Ground-based observations of Saturn's North Polar Spot and Hexagon. Science, 260, 329-332.

Sánchez Lavega, A., J. F. Rojas, J. R. Acarreta, J. Lecacheux, F. Colas, and P. V. Sada, 1997. New observations and studies of Saturn's long-lived North Polar Spot, Icarus, $128,322-334$. 
Sánchez-Lavega, A., R. Hueso, S. Pérez-Hoyos, and J. F. Rojas, 2006. A strong vortex in Saturn's south pole.Icarus 184, 524-531.

Sánchez-Lavega, A., T. del Río-Gaztelurrutia, R. Hueso, J. M. Gomez-Forrellad, J. F. Sanz-Requena, J. Legarreta, E. García-Melendo, F. Colas, J. Lecacheux, L. N. Fletcher, D. Barrado-Navascués, D. Parker \& the International Outer Planet Watch Team,T. Akutsu, T. Barry, J. Beltran, S. Buda, B. Combs, F. Carvalho, P. Casquinha, M. Delcroix, S. Ghomizadeh, C. Go, J. Hotershall, T. Ikemura, G. Jolly, A. Kazemoto, T. Kumamori, M. Lecompte, P. Maxson, F. J. Melillo, D. P. Milika, E. Morales, D. Peach, J. Phillips, J. J. Poupeau, J. Sussenbach, G. Walker, S. Walker, T. Tranter, A. Wesley, T. Wilson \& K. Yunoki for The International Outer Planet Watch (IOPW) Team, 2011 . Deep winds beneath Saturn's upper clouds from a seasonal long-lived planetary-scale storm. Nature 475, 71-74 (2011);doi:10.1038/nature10203.

Sánchez-Lavega, A., 2011. An Introduction to Planetary Atmospheres.CRC Press, Boca Raton, FL, USA.

Sánchez-Lavega, A., Río-Gaztelurrutia, T., Hueso, R., et al., 2014. The long-term steady motion of Saturn's Hexagon and the stability of its enclosed jet stream under seasonal changes.GeophysicalResearchLetters, 41 (Mar.), 1425-1431.

Sánchez-Lavega A., D. Garcia, T. del Rio-Gaztelurrutia, A. Garcia-Muñoz, S. PerezHoyos, R. Hueso, 2017. Cassini limb images of hazes in Saturn's northern hemisphere, 49th DPS meeting, abstract 205.6.

Sayanagi, K. M., S. P. Ewald, U. A. Dyudina, and A. P. Ingersoll, 2013. American Astronomical Society, DPS meeting \#45, \#509.06.10.

Sayanagi, K.M., Kevin H. Baines, Ulyana A. Dyudina, Leigh N. Fletcher, Agustin Sánchez-Lavega, Robert A. West, 2016. Saturn's polar atmosphere. Chapter for Saturn in the 21st Century.

Sayanagi, K.M., Blalock, J.J, Dyudina, U.A., Ewald, S.P., Ingersoll, A.P., 2017. Cassini ISS observation of Saturn's north polar vortes comparison to the south polar vortex. Icarus 285, 68-82.

Sylvestre, M., Guerlet, S., Fouchet,nT. Spiga, A., Flasar, F.M., Hesman, B., Bjoraker, G.L.2015 Seasonal changes in Saturn's stratosphere inferred from Cassini/CIRS limb observations. Icarus, 258, 224-238.

Tomasko, M.G., Doose, L.R., 1984. Polarimetry and photometry of Saturn from Pioneer 11: Observations and constraints on the distribution and properties of cloud and aerosol particles. Icarus 58, 1-34.

West, R.A., Smith, P.H., 1991. Evidence for aggregate particles in the atmospheres of Titan and Jupiter. Icarus 90, 330-333.

West,R.A., Baines,K.H., Karkoschka, E., Sánchez-Lavega, A., 2009. Clouds and aerosols in Saturn's atmosphere. In: Dougherty, M.K., Esposito, L.W., Krimigis, S.M.(Eds.), Saturn from Cassini-Huygens. Springer, Netherlands, pp.161-179. 
904 West, R. et al., 2010. In-flight calibration of the Cassin imaging sciencesub-system 905 camera.Planet. Space Sci.58, 1475-1488.

906 West, R.A., P.A. Yanamandra-Fisher and V. Korokhin, 2015. Polarization of the gas 907 giant planets, Saturn's rings, and Titan: Observations and Interpretation", in Polarimetry 908 of stars and planetary systems, L. Kolokolva, J. Hough and A.-Chantal Levasseur909 Regourd Eds., Cambridge Univ. Press. 